\title{
Random Matrices with Merging Singularities and the Painlevé V Equation ${ }^{\star}$
}

\author{
Tom CLAEYS and Benjamin FAHS
}

Institut de Recherche en Mathématique et Physique, Université catholique de Louvain, Chemin du Cyclotron 2, B-1348 Louvain-La-Neuve, Belgium

E-mail:tom.claeys@uclouvain.be,benjamin.fahs@uclouvain.be

Received September 08, 2015, in final form March 18, 2016; Published online March 23, 2016

http://dx.doi.org/10.3842/SIGMA.2016.031

\begin{abstract}
We study the asymptotic behavior of the partition function and the correlation kernel in random matrix ensembles of the form $\frac{1}{Z_{n}}\left|\operatorname{det}\left(M^{2}-t I\right)\right|^{\alpha} e^{-n \operatorname{Tr} V(M)} d M$, where $M$ is an $n \times n$ Hermitian matrix, $\alpha>-1 / 2$ and $t \in \mathbb{R}$, in double scaling limits where $n \rightarrow \infty$ and simultaneously $t \rightarrow 0$. If $t$ is proportional to $1 / n^{2}$, a transition takes place which can be described in terms of a family of solutions to the Painlevé $\mathrm{V}$ equation. These Painlevé solutions are in general transcendental functions, but for certain values of $\alpha$, they are algebraic, which leads to explicit asymptotics of the partition function and the correlation kernel.
\end{abstract}

Key words: random matrices; Painlevé equations; Riemann-Hilbert problems

2010 Mathematics Subject Classification: 60B20; 35Q15; 33E17

Dedicated to Percy Deift and Craig Tracy on the occasion of their 70th birthdays.

\section{Introduction}

We consider a random matrix model on $n \times n$ Hermitian matrices, defined by a probability measure of the form

$$
\frac{1}{Z_{n}}\left|\operatorname{det}\left(M^{2}-t I\right)\right|^{\alpha} e^{-n \operatorname{Tr} V(M)} d M \quad \text { for } \quad \alpha>-1 / 2, \quad t \in \mathbb{R} .
$$

Here $d M=\prod_{1 \leq i \leq n} d M_{i i} \prod_{1 \leq i<j \leq n} d \operatorname{Re} M_{i j} d \operatorname{Im} M_{i j}$ is the Lebesgue measure on $n \times n$ Hermitian matrices and $Z_{n}=Z_{n}(t, \alpha, V)$ is a normalizing constant. The confining potential $V: \mathbb{R} \rightarrow \mathbb{R}$ is a real analytic function which increases sufficiently fast asymptotically, namely

$$
\lim _{x \rightarrow \pm \infty} \frac{V(x)}{\log \left(x^{2}+1\right)}=+\infty
$$

The measure (1.1) is invariant under unitary conjugation and induces a probability measure on the eigenvalues $x_{i}, i=1,2, \ldots, n$, given by

$$
\frac{1}{\widehat{Z}_{n}} \prod_{1 \leq i<j \leq n}\left(x_{j}-x_{i}\right)^{2} \prod_{j=1}^{n} w_{n}\left(x_{j}\right) d x_{j}, \quad w_{n}(x)=e^{-n V(x)}\left|x^{2}-t\right|^{\alpha},
$$

${ }^{\star}$ This paper is a contribution to the Special Issue on Asymptotics and Universality in Random Matrices, Random Growth Processes, Integrable Systems and Statistical Physics in honor of Percy Deift and Craig Tracy. The full collection is available at http://www.emis.de/journals/SIGMA/Deift-Tracy.html 
where

$$
\widehat{Z}_{n}=\widehat{Z}_{n}(t, \alpha, V)=\int_{-\infty}^{\infty} \cdots \int_{-\infty}^{\infty} \prod_{1 \leq i<j \leq n}\left(x_{j}-x_{i}\right)^{2} \prod_{j=1}^{n} w_{n}\left(x_{j}\right) d x_{j}
$$

is the partition function associated to the ensemble. It can alternatively be written as the Hankel determinant

$$
\widehat{Z}_{n}(t, \alpha, V)=n ! \operatorname{det}\left(\int_{-\infty}^{+\infty} x^{i+j} w_{n}(x) d x\right)_{i, j=0, \ldots, n-1} .
$$

While $\alpha$ is kept fixed and does not depend on the matrix size $n$, we will be particularly interested in double scaling limits where $t$ is $n$-dependent and approaches 0 as $n \rightarrow \infty$. In such a case, the weight function $w_{n}$ has the feature that it has two merging singularities approaching 0 as $n \rightarrow \infty$. If $t>0$, the two singularities $\pm \sqrt{t}$ are real; if $t<0$, the singularities $\pm i \sqrt{-t}$ are purely imaginary. Random matrix ensembles with such merging root-type singularities have applications in fractional Brownian motion with Hurst index $H=0$ [19], and in quantum chromodynamics $[1,6]$. The partition function $\widehat{Z}_{n}$ appears in the study of impenetrable bosons in a harmonic well as the one body density matrix (up to a constant) [17].

Although the singularities have no effect on the macroscopic limiting density of the eigenvalues as $n \rightarrow \infty$, they do have an effect on the partition function $\widehat{Z}_{n}$ and on the microscopic correlations between eigenvalues near the origin. We will obtain asymptotics for the partition function and for the eigenvalue correlation kernel near the origin, in double scaling limits where $n \rightarrow \infty$ and $t \rightarrow 0$ simultaneously. The asymptotics for the partition function are described in terms of solutions to the fifth Painlevé equation; the limit of the correlation kernel can also be expressed in terms of functions related to the Painlevé $\mathrm{V}$ equation. As $t \rightarrow 0$ at a fast rate, the limiting kernel degenerates to a Bessel kernel, and as $t \rightarrow 0$ at a slow rate, we recover the sine kernel.

\subsection{Statement of results}

The macroscopic large $n$ behavior of the eigenvalues is described by the limit of the mean eigenvalue distribution as $n \rightarrow \infty$, which we denote by $\mu_{V}$, and which is independent of $t$ and $\alpha$, for $t, \alpha$ bounded. It is characterized $[7,10]$ as the unique equilibrium measure $\mu_{V}$ which minimizes

$$
\iint \log \frac{1}{|x-y|} d \mu(x) d \mu(y)+\int V(x) d \mu(x),
$$

over the space of Borel probability measures $\mu$ on $\mathbb{R}$. The equilibrium measure is alternatively characterized by the Euler-Lagrange variational conditions [30]

$$
\begin{aligned}
& 2 \int \log |x-y| d \mu_{V}(y)=V(x)-\ell, \quad \text { for } \quad x \in \operatorname{supp} \mu_{V}, \\
& 2 \int \log |x-y| d \mu_{V}(y) \leq V(x)-\ell, \quad \text { for } \quad x \in \mathbb{R} \backslash \operatorname{supp} \mu_{V},
\end{aligned}
$$

for some constant $\ell$ depending on $V$.

Throughout the paper we will assume that $V$ is such that the equilibrium measure is supported on a single interval $[a, b]$. Then, the equilibrium measure can be written in the form [10]

$$
d \mu_{V}(x)=\psi_{V}(x) d x=\sqrt{(b-x)(x-a)} h(x) d x, \quad x \in[a, b],
$$

with $h$ a real analytic function. We require $V$ to be such that the following generic conditions hold: the density $\psi_{V}$ is strictly positive on $(a, b)$, it vanishes like a square root at the endpoints $a, b$ (in other words, $h(x)>0$ for $x \in[a, b]$ ), and the variational inequality (1.8) is strict 
on $\mathbb{R} \backslash[a, b]$. If all those assumptions hold, we say that $V$ is one-cut regular (see [26] for a general classification of singularities). In addition, we require zero to be contained in the interior of the support of $\mu_{V}$, i.e., $a<0<b$.

If zero were outside of the support of $\mu_{V}$, no eigenvalues are expected near the origin for large $n$, and the singularities of the weight will not play a significant role for large $n$. The case where 0 is an edge point of the support of $V$ is also interesting, but will not be studied here - we refer to [22] for results about the local eigenvalue correlations when a singularity lies near a soft edge, and to [33] when a singularity approaches a hard edge in a modified Jacobi ensemble.

\section{Painlevé V functions}

In order to describe the asymptotic behavior in the matrix model (1.1) for $n$ large and $t$ small, we need a special 1-parameter form of the more general 4-parameter Jimbo-Miwa-Okamoto $\sigma$-form of the fifth Painlevé equation (see [23, 24], and [18] for the explicit relation to the usual Painlevé $\mathrm{V}$ equation), which is given by

$$
\left(s \sigma^{\prime \prime}(s)\right)^{2}=\left(\sigma(s)-s \sigma^{\prime}(s)+2\left(\sigma^{\prime}(s)\right)^{2}+2 \alpha \sigma^{\prime}(s)\right)^{2}-4\left(\sigma^{\prime}(s)\right)^{2}\left(\sigma^{\prime}(s)+\alpha\right)^{2} .
$$

For $\alpha>-1 / 2$, we are interested in two particular solutions $\sigma_{\alpha}^{+}$and $\sigma_{\alpha}^{-}$to this equation. The first one, $\sigma_{\alpha}^{-}(s)$, is analytic and real for $s \in(0,+\infty)$, and it has the asymptotics

$$
\sigma_{\alpha}^{-}(s)= \begin{cases}\alpha^{2}+o(1), & s \rightarrow 0 \\ s^{-1+2 \alpha} e^{-s} \frac{1}{\Gamma(\alpha)^{2}}\left(1+\mathcal{O}\left(s^{-1}\right)\right), & s \rightarrow+\infty\end{cases}
$$

where $\Gamma$ is Euler's $\Gamma$-function. The existence of such a solution was proved in [4]. The second solution $\sigma_{\alpha}^{+}(s)$ is analytic and such that $\sigma_{\alpha}^{+}(s)+\frac{\alpha s}{2}$ is real-valued for $s \in-i \mathbb{R}_{>0}$, satisfying in addition:

$$
\sigma_{\alpha}^{+}(s)= \begin{cases}\alpha^{2}+o(1), & s \rightarrow-i 0^{+}, \\ \frac{\alpha^{2}}{2}-\frac{\alpha s}{2}+\mathcal{O}\left(|s|^{-1}\right), & s \rightarrow-i \infty .\end{cases}
$$

Its existence was proved in [5]. It should be noted that uniqueness of the solutions $\sigma_{\alpha}^{ \pm}$satisfying the above asymptotic conditions was not proven in [4, 5]. Those Painlevé solutions $\sigma_{\alpha}^{-}$and $\sigma_{\alpha}^{+}$ can be characterized in terms of Riemann-Hilbert $(\mathrm{RH})$ problems; we will do this in Section 2 for $\sigma_{\alpha}^{-}$and in Section 3 for $\sigma_{\alpha}^{+}$. For general values of $\alpha>-1 / 2, \sigma_{\alpha}^{ \pm}$are transcendental functions, but there are special values of $\alpha$ where they are algebraic. For $\sigma_{\alpha}^{-}$, this is the case if $\alpha$ is an integer, while for $\sigma_{\alpha}^{+}$only if $\alpha$ is an even integer. For those values of $\alpha, \sigma_{\alpha}^{ \pm}$can be constructed via a recursive procedure, involving Schlesinger type transformations [14], which we will explain in detail in Sections 2.2 and 3.2.

For $\alpha=0,1,2$, the expressions are simple and we have

$$
\begin{aligned}
& \sigma_{0}^{ \pm}(s)=0, \\
& \sigma_{1}^{-}(s)=\frac{s}{e^{s}-1}, \\
& \sigma_{2}^{ \pm}(s)=s \frac{-2+e^{s}\left(2-2 s+s^{2}\right)}{1-e^{s}\left(2+s^{2}\right)+e^{2 s}} .
\end{aligned}
$$

\section{Asymptotics for the partition function}

Theorem 1.1. Let $V$ be real analytic, satisfying (1.2) and one-cut regular with 0 in the interior of the support $[a, b]$ of the equilibrium measure $\mu_{V}$. Denote $z_{0}=\sqrt{t}$ when $t$ is positive and 
$z_{0}=i \sqrt{-t}$ when $t$ is negative. Define

$$
s_{n, t}=-2 \pi i n \int_{-z_{0}}^{z_{0}} h(s)((s-a)(b-s))^{1 / 2} d s,
$$

with $h$ defined in (1.9) and where the square root is taken to be positive at 0 and analytic in a neighbourhood of 0 , in such a way that $s_{n, t}$ is positive when $t<0$ and negative imaginary when $t>0$. We assume that $\alpha>-1 / 2$ and $t<0$ or that $\alpha>0$ and $t>0$. Then, the partition function $\widehat{Z}_{n}(t)$ defined in (1.4) satisfies

$$
\begin{aligned}
\log \widehat{Z}_{n}(t, \alpha, V)= & \log \widehat{Z}_{n}(0, \alpha, V)+\int_{0}^{s_{n, t}} \frac{\sigma_{\alpha}^{ \pm}(s)-\alpha^{2}}{s} d s+\frac{\alpha s_{n, t}}{2} \\
& +\frac{n \alpha}{2}\left(V\left(z_{0}\right)+V\left(-z_{0}\right)-2 V(0)\right)+\mathcal{O}\left(|t|^{1 / 2}\right)+\mathcal{O}\left(n^{-1}\right),
\end{aligned}
$$

with uniform error terms as $n \rightarrow \infty$ and $t \rightarrow 0, \pm t>0$.

Remark 1.2. If we set $\widehat{s}_{n, t}=-4 \pi i n z_{0} \psi_{V}(0)$, we have $\widehat{s}_{n, t}=s_{n, t}(1+\mathcal{O}(|t|))$ as $n \rightarrow \infty, t \rightarrow 0$. If $t>0$, it follows from equation (1.36) below that one can replace $s_{n, t}$ by $\widehat{s}_{n, t}$ in (1.15) without modifying the error terms. Doing the same when $t<0$ induces an error term of order $\mathcal{O}\left(n|t|^{3 / 2}\right)$.

Remark 1.3. We believe that the expansion (1.15), with error term $o(1)$, holds for $t>0$ and $-1 / 2<\alpha<0$ as well, but because of technical obstacles, we do not prove the result in this case.

Remark 1.4. The asymptotic behavior for $\widehat{Z}_{n}(t, \alpha, V)$ is described in terms of the Painlevé V solutions $\sigma_{\alpha}^{ \pm}$and in terms of the partition function $\widehat{Z}_{n}(0, \alpha, V)$, which corresponds to a weight function which has only one singularity at 0 . Asymptotics for $\widehat{Z}_{n}(0, \alpha, V)$ were obtained in [25] in the case where $V$ is quadratic, an important special case which we will return to later in this section. For general $V$, we have not been able to find explicit asymptotic expansions in the literature for $\log \widehat{Z}_{n}(0, \alpha, V)$, up to decaying terms as $n \rightarrow \infty$.

Remark 1.5. By (1.12)-(1.14), the integral appearing in (1.15) can be computed explicitly for $\alpha=0,1,2$. We have

$$
\begin{aligned}
& \int_{0}^{s_{n, t}} \frac{\sigma_{0}^{ \pm}(s)}{s} d s=0, \\
& \int_{0}^{s_{n, t}} \frac{\sigma_{1}^{-}(s)-1}{s} d s=\log \left(2 \sinh \frac{s_{n, t}}{2}\right)-\frac{s_{n, t}}{2}-\log s_{n, t}, \\
& \int_{0}^{s_{n, t}} \frac{\sigma_{2}^{ \pm}(s)-4}{s} d s=\log \left(4 \sinh ^{2} \frac{s_{n, t}}{2}-s_{n, t}^{2}\right)-s_{n, t}-4 \log s_{n, t}+\log 12 .
\end{aligned}
$$

Remark 1.6. Our proofs of Theorems 1.1 and 1.7 are based on an asymptotic analysis of the orthogonal polynomials on the real line defined by (1.19), using RH methods [7, 11, 12]. The asymptotic analysis for those orthogonal polynomials shows similarities to the one performed in $[4,5]$ (following the general method from [8]) for orthogonal polynomials on the unit circle with respect to weights with merging or emerging singularities, where they were used to obtain asymptotics for Toeplitz determinants. Nevertheless, there is no known way to directly derive the asymptotics for the Hankel determinant (1.5), with respect to the $n$-dependent weight $w_{n}$ supported on the full real line, from the asymptotics for Toeplitz determinants.

\section{Asymptotics for the correlation kernel near 0}

Denote by $p_{j}^{(n)}$ the degree $j$ orthonormal polynomials with respect to the weight $w_{n}(x)$ (defined in (1.3)), characterized by

$$
\int_{\mathbb{R}} p_{j}^{(n)}(x) p_{k}^{(n)} w_{n}(x) d x=\delta_{j k}= \begin{cases}0 & \text { for } j \neq k \\ 1 & \text { for } j=k,\end{cases}
$$


with positive leading coefficient $\kappa_{j}^{(n)}$. The eigenvalue density (1.3) characterizes a determinantal point process with correlation kernel given by

$$
K_{n}(x, y)=\sqrt{w_{n}(x) w_{n}(y)} \sum_{j=0}^{n-1} p_{j}^{(n)}(x) p_{j}^{(n)}(y),
$$

or alternatively

$$
K_{n}(x, y)=\sqrt{w_{n}(x) w_{n}(y)} \frac{\kappa_{n-1}^{(n)}}{\kappa_{n}^{(n)}} \frac{p_{n}^{(n)}(x) p_{n-1}^{(n)}(y)-p_{n-1}^{(n)}(x) p_{n}^{(n)}(y)}{x-y},
$$

by the Christoffel-Darboux formula. Note that the orthogonal polynomials and the correlation kernel $K_{n}$ depend on the parameter $t$ in the weight $w_{n}$. This paper is concerned with the microscopic large $n$ behavior of the eigenvalues near 0 . We will study the scaled correlation kernel $\frac{1}{c n} K_{n}\left(\frac{u}{c n}, \frac{v}{c n}\right)$ for a suitable choice of $c>0$, and obtain asymptotics for it as $n \rightarrow \infty$ and $t \rightarrow 0$. We will see that a transition in the asymptotics for the kernel takes place if $t$ is of the order $n^{-2}$, or equivalently if the distance between the two singularities in the weight is of the order $n^{-1}$, which is of the same order as the typical distance between consecutive eigenvalues.

Local scaling limits near the origin for the correlation kernel $K_{n}$ are well understood for $t \neq 0$ fixed and for $t=0$, as $n \rightarrow \infty$. If $t \neq 0$ is independent of $n$, then, as a slight generalization of results obtained in $[11,12]$, we have for $u, v \in \mathbb{R}$ that

$$
\lim _{n \rightarrow \infty} \frac{1}{\psi_{V}(0) n} K_{n}\left(\frac{u}{\psi_{V}(0) n}, \frac{v}{\psi_{V}(0) n}\right)=\mathbb{K}^{\sin }(u, v)=\frac{\sin \pi(u-v)}{\pi(u-v)} .
$$

The convergence is uniform for $u, v$ in compact subsets of $\mathbb{R}$. If $t=0$, it was proved in [27] that for $u, v$ in bounded subsets of $(0, \infty)$ that

$$
\begin{aligned}
\lim _{n \rightarrow \infty} & \frac{1}{\psi_{V}(0) n} K_{n}\left(\frac{u}{\psi_{V}(0) n}, \frac{v}{\psi_{V}(0) n}\right)=\mathbb{K}_{\alpha}^{\mathrm{Bessel}}(u, v) \\
& =\pi \sqrt{u v} \frac{J_{\alpha+\frac{1}{2}}(\pi u) J_{\alpha-\frac{1}{2}}(\pi v)-J_{\alpha-\frac{1}{2}}(\pi u) J_{\alpha+\frac{1}{2}}(\pi v)}{2(u-v)},
\end{aligned}
$$

where $J_{\nu}$ is the Bessel function of order $\nu$ (see [29] for a reference on Bessel functions and other special functions which appear throughout the paper). The convergence is uniform for $u, v$ in compact subsets of $(0, \infty)$.

We obtain large $n$ asymptotics for the correlation kernel near the origin as $n \rightarrow \infty$ for $t$ small. Of particular interest will be the double scaling limit where $n \rightarrow \infty$ and simultaneously $t \rightarrow 0$ in such a way that $n^{2} t$ tends to a non-zero constant. This will lead us to a new family of limiting kernels which are built out of functions associated to the Painlevé V equation.

Theorem 1.7. Let $V$ be real analytic, satisfying (1.2) and one-cut regular with 0 in the interior of the support $[a, b]$ of the equilibrium measure $\mu_{V}$. We denote $\psi_{V}$ for the density of $\mu_{V}$, given by (1.9), and define

$$
\tau_{n, t}=16 \pi^{2} \psi_{V}(0)^{2} n^{2} t
$$

for $t$ real.

1. As $n \rightarrow \infty$ and simultaneously $t \rightarrow 0$ in such a way that $\tau_{n, t} \rightarrow \pm \infty$, we have

$$
\lim \frac{1}{\psi_{V}(0) n} K_{n}\left(\frac{u}{\psi_{V}(0) n}, \frac{v}{\psi_{V}(0) n}\right)=\mathbb{K}^{\sin }(u, v),
$$

for any $u, v \in \mathbb{R}$, with $\mathbb{K}^{\text {sin }}$ as in (1.21). 
2. As $n \rightarrow \infty$ and simultaneously $t \rightarrow 0$ in such a way that $\tau_{n, t} \rightarrow 0$, we have

$$
\lim \frac{1}{\psi_{V}(0) n} K_{n}\left(\frac{u}{\psi_{V}(0) n}, \frac{v}{\psi_{V}(0) n}\right)=\mathbb{K}_{\alpha}^{\text {Bessel }}(u, v),
$$

for any $u, v \in \mathbb{R} \backslash\{0\}$, with $\mathbb{K}_{\alpha}^{\text {Bessel }}$ as in (1.22).

3. As $n \rightarrow \infty$ and simultaneously $t \rightarrow 0$ in such a way that $\tau_{n, t} \rightarrow \tau \neq 0$, there exists a limiting kernel $\mathbb{K}_{\alpha}^{\mathrm{PV}}$ such that

$$
\lim \frac{1}{\psi_{V}(0) n} K_{n}\left(\frac{u}{\psi_{V}(0) n}, \frac{v}{\psi_{V}(0) n}\right)=\mathbb{K}_{\alpha}^{\mathrm{PV}}(u, v ; \tau),
$$

for any $u, v \in \mathbb{R}$ if $\tau<0$, and for any $u, v \in \mathbb{R} \backslash\{ \pm \sqrt{\tau} / 4\}$ if $\tau>0$.

The limits are uniform for $u, v$ in compact subsets of $\mathbb{R}$.

Remark 1.8. The limiting kernel $\mathbb{K}_{\alpha}^{\mathrm{PV}}(u, v ; \tau)$ has the form

$$
\mathbb{K}_{\alpha}^{\mathrm{PV}}(u, v ; \tau)=\frac{\Phi_{1}(\pi v ; \tau) \Phi_{2}(\pi u ; \tau)-\Phi_{1}(\pi u ; \tau) \Phi_{2}(\pi v ; \tau)}{2 \pi i(u-v)},
$$

and the functions $\Phi_{j}, j=1,2$, will be defined in Section 2 for $\tau<0$ and in Section 3 for $\tau>0$. The easiest way to define $\Phi_{1}$ and $\Phi_{2}$ is via a $\mathrm{RH}$ problem which appeared in [4,5]. Alternatively, they can be characterized as special solutions to a Lax pair which is related to the fifth Painlevé equation. For $\tau<0, \Phi_{1}(u ; \tau)$ and $\Phi_{2}(u ; \tau)$ are analytic as functions of $u$ in a neighborhood of $\mathbb{R}$; for $\tau>0$, they are analytic functions of $u$ in a neighborhood of $\mathbb{R} \backslash\left\{ \pm \frac{\sqrt{\tau}}{4}\right\}$; they are analytic as functions of $\tau$ for $\tau$ in a neighborhood of $\mathbb{R} \backslash\{0\}$ and continuous in $\tau$ at 0 . For $\tau=0$, the limiting kernel $\mathbb{K}_{\alpha}^{\mathrm{PV}}$ is a special case of a large family of limiting kernels which appeared in [2].

Remark 1.9. In general, the kernel $\mathbb{K}_{\alpha}^{\mathrm{PV}}$ is a transcendental function of $u, v, \tau$. However, for $\tau<0$ and $\alpha \in \mathbb{N}$, and for $\tau>0$ and $\alpha$ even, the kernel $\mathbb{K}_{\alpha}^{\mathrm{PV}}$ is algebraic and can be constructed explicitly by a recursive procedure. We will present this procedure in Section 2.2 for $\tau<0$ and in Section 3.2 for $\tau>0$.

Remark 1.10. The limiting kernel $\mathbb{K}_{\alpha}^{\mathrm{PV}}(u, v ; \tau)$ degenerates to the sine and Bessel kernels for large and small values of $\tau$. Indeed, we will show that

$$
\lim _{\tau \rightarrow \pm \infty} \mathbb{K}_{\alpha}^{\mathrm{PV}}(u, v ; \tau)=\mathbb{K}^{\sin }(u, v), \quad u, v \in \mathbb{R}
$$

and that

$$
\lim _{\tau \rightarrow 0} \mathbb{K}_{\alpha}^{\mathrm{PV}}(u, v ; \tau)=\mathbb{K}_{\alpha}^{\mathrm{Bessel}}(u, v), \quad u, v \in \mathbb{R} \backslash\{0\} .
$$

This implies that (1.24), (1.25), and (1.26) are consistent: letting $\tau \rightarrow \pm \infty$ in (1.26), we recover (1.24), and letting $\tau \rightarrow 0$, we get (1.25).

\subsection{Consequences and applications}

\section{Asymptotics for Toeplitz determinants}

In [4], large $n$ asymptotics were obtained for Toeplitz determinants

$$
D_{n}(f)=\operatorname{det}\left(f_{j-k}\right)_{j, k=0}^{n-1}, \quad f_{j}=\frac{1}{2 \pi} \int_{0}^{2 \pi} f\left(e^{i \theta}\right) e^{-i j \theta} d \theta
$$


with an emerging Fisher-Hartwig singularity, depending on parameters $\alpha, \beta$. In the special case $\beta=0$, the weight has the form

$$
f_{t}(z)=\left(z-e^{t}\right)^{\alpha}\left(z-e^{-t}\right)^{\alpha} z^{-\alpha} e^{-\pi i \alpha} e^{V(z)}, \quad z=e^{i \theta}, \quad \theta \in[0,2 \pi)
$$

for $\operatorname{Re} \alpha>-\frac{1}{2}, t>0$, and where $V(z)=\sum_{k=-\infty}^{+\infty} V_{k} z^{k}$ is analytic on an annulus containing the unit circle. The singularities $e^{ \pm t}$ approach the unit circle as $t \rightarrow 0$, and form a Fisher-Hartwig type singularity if $t=0$. Asymptotics for Toeplitz determinants with weight functions of this form were also obtained in the context of the $2 \mathrm{~d}$ Ising model [32], see [9] for a review of Toeplitz determinants and the Ising model. Theorem 1.1 in [4] states that

$$
\begin{aligned}
\log D_{n}\left(f_{t}\right)= & n V_{0}+\alpha n t+\sum_{k=1}^{\infty} k\left(V_{k}-\alpha \frac{e^{-t k}}{k}\right)\left(V_{-k}-\alpha \frac{e^{-t k}}{k}\right) \\
& +\log \frac{G(1+\alpha)^{2}}{G(1+2 \alpha)}+\alpha^{2} \log 2 n t+\int_{0}^{2 n t} \frac{\sigma_{\alpha}^{-}(x)-\alpha^{2}}{x} d x+o(1),
\end{aligned}
$$

as $n \rightarrow \infty$ and simultaneously $t \rightarrow 0$ where $G$ is Barnes's G-function. If $\beta=0$ and $\alpha=1$, we we can substitute (1.17) and (1.18), here with $s_{n, t}=2 n t$, and obtain the more explicit asymptotic expansions

$$
\log D_{n}\left(f_{t}\right)=n V_{0}+\sum_{k=1}^{\infty} k V_{k} V_{-k}-\sum_{k=1}^{\infty}\left(V_{k}+V_{-k}\right) e^{-t k}+\log \frac{\sinh n t}{\sinh t}+t+o(1),
$$

for $\alpha=1$, and

$$
\begin{aligned}
\log D_{n}\left(f_{t}\right)= & n V_{0}+\sum_{k=1}^{\infty} k V_{k} V_{-k}-2 \sum_{k=1}^{\infty}\left(V_{k}+V_{-k}\right) e^{-t k}+4 t \\
& -2 \log \left(2 \sinh ^{2} t\right)+\log \left(\sinh ^{2} n t-n^{2} t^{2}\right)+o(1)
\end{aligned}
$$

for $\alpha=2$, as $n \rightarrow \infty$ and $t \rightarrow 0$. With more effort, we can obtain explicit asymptotic expansions for any integer $\alpha$.

In [5], asymptotics were obtained for Toeplitz determinants $D_{n}\left(f_{t}\right)$ with merging FisherHartwig singularities, depending on parameters $\alpha_{1}, \alpha_{2}, \beta_{1}, \beta_{2}$. Setting $\alpha_{1}=\alpha_{2}=\alpha / 2$ and $\beta_{1}=\beta_{2}=0$, the weight has the form

$$
f_{t}(z)=e^{V(z)}\left|z-e^{i t}\right|^{\alpha}\left|z-e^{i(2 \pi-t)}\right|^{\alpha},
$$

where $\operatorname{Re} \alpha>-\frac{1}{2}, t \in(0, \pi)$, and with $V$ again analytic on an annulus containing the unit circle. Then, the weight function has two Fisher-Hartwig type singularities if $t>0$ which merge to a single one as $t \rightarrow 0$. In [5, Theorem 1.5], the following result is stated:

$$
\begin{aligned}
\log D_{n}\left(f_{t}\right)= & \log D_{n}\left(f_{0}\right)+\int_{0}^{-2 i n t} \frac{1}{s}\left(\sigma_{\mathrm{CK}}(s)-\frac{\alpha^{2}}{2}\right) d s \\
& -\frac{\alpha^{2}}{2} \log \frac{\sin t}{t}-\frac{\alpha}{2}\left(V\left(e^{i t}\right)+V\left(e^{-i t}\right)-2 V(1)\right)+o(1)
\end{aligned}
$$

as $n \rightarrow \infty$ and $t \rightarrow 0$. The function $\sigma_{\mathrm{CK}}$ is related to $\sigma_{\alpha}^{+}$by the formula

$$
\sigma_{\mathrm{CK}}(s)=\sigma_{\alpha}^{+}(s)-\frac{\alpha^{2}}{2}+\frac{\alpha s}{2}
$$


For $\alpha=2$, we can substitute (1.18) (with $s_{n, t}=-2 i n t$ ) and obtain

$$
\begin{aligned}
\log D_{n}\left(f_{t}\right)= & n V_{0}+\sum_{k=1}^{\infty} k V_{k} V_{-k}-2 \log 2 t \sin t+\log \left(n^{2} t^{2}-\sin ^{2} n t\right) \\
& -\left(V\left(e^{i t}\right)+V\left(e^{-i t}\right)-2 V_{0}\right)+o(1),
\end{aligned}
$$

as $n \rightarrow \infty$ and $t \rightarrow 0$. With more effort, we can obtain explicit asymptotic expansions for any even $\alpha$.

\section{Extreme values of GUE characteristic polynomials}

Let $H$ be a random $n \times n$ GUE matrix, normalized such that the joint probability distribution of the eigenvalues is given by

$$
\frac{1}{\widehat{Z}_{n}^{\mathrm{GUE}}} \prod_{1 \leq i<j \leq n}\left(x_{j}-x_{i}\right)^{2} \prod_{j=1}^{n} e^{-2 n x_{j}^{2}} d x_{j}
$$

where $\widehat{Z}_{n}^{\mathrm{GUE}}=Z_{n}\left(\alpha=0, V(x)=2 x^{2}\right)$ is the normalizing constant. The limiting mean eigenvalue density is then supported on $[-1,1]$ as $n \rightarrow \infty$. Define the (random) characteristic polynomial

$$
P_{n}(x)=\operatorname{det}(x I-H) .
$$

The average of products of powers of characteristic polynomials of the form $\mathbb{E}\left(\prod_{j=1}^{2}\left|P_{n}\left(u_{j}\right)\right|^{\alpha}\right)$, given $u_{1}, u_{2} \in \mathbb{R}$, can be expressed as

$$
\mathbb{E}\left(\prod_{j=1}^{2}\left|P_{n}\left(u_{j}\right)\right|^{\alpha}\right)=\frac{1}{\widehat{Z}_{n}^{\mathrm{GUE}}} \int_{\mathbb{R}^{n}} \prod_{1 \leq i<j \leq n}\left(x_{j}-x_{i}\right)^{2} \prod_{j=1}^{n}\left|x_{j}-u_{1}\right|^{\alpha}\left|x_{j}-u_{2}\right|^{\alpha} e^{-2 n x_{j}^{2}} d x_{j} .
$$

The following asymptotic results as $n \rightarrow \infty$ were obtained by Krasovsky [25] (see also [3, 16, 21] for $\alpha_{j}$ 's integers):

$$
\mathbb{E}\left(\prod_{j=1}^{k}\left|P_{n}\left(u_{j}\right)\right|^{2 \alpha_{j}}\right)=F\left(n,\left(u_{i}, \alpha_{i}\right)_{i=1}^{k}\right)\left(1+\mathcal{O}\left(\frac{\log n}{n}\right)\right),
$$

where $k=1,2$ and

$$
\begin{aligned}
& F\left(n,\left(u_{i}, \alpha_{i}\right)_{i=1}^{k}\right)=\prod_{j=1}^{k} C\left(\alpha_{j}\right)\left(1-u_{j}^{2}\right)^{\alpha_{j}^{2} / 2}(n / 2)^{\alpha_{j}^{2}} e^{\left(2 u_{j}^{2}-1-2 \log 2\right) \alpha_{j} n} \prod_{1 \leq i<j \leq k}\left(2\left|u_{i}-u_{j}\right|\right)^{-2 \alpha_{i} \alpha_{j}}, \\
& C(\alpha)=2^{2 \alpha^{2}} \frac{G(\alpha+1)^{2}}{G(2 \alpha+1)}
\end{aligned}
$$

and $G$ is the Barnes G-function. If $u_{1}$ and $u_{2}$ approach each other, we can use Theorem 1.1 to obtain asymptotics for $\mathbb{E}\left(\prod_{j=1}^{2}\left|P_{n}\left(u_{j}\right)\right|^{2 \alpha_{j}}\right)$. Indeed, by (1.30) and (1.4), it follows that

$$
\mathbb{E}\left(\prod_{j=1}^{2}\left|P_{n}\left(u_{j}\right)\right|^{\alpha}\right)=\frac{\widehat{Z}_{n}\left(t=\frac{\left(u_{1}-u_{2}\right)^{2}}{4}, \alpha, V(x)=2\left(x+\left(u_{1}+u_{2}\right) / 2\right)^{2}\right)}{\widehat{Z}_{n}^{\mathrm{GUE}}},
$$


which is seen to hold after the simple change of variables $y_{n}=x_{n}-\frac{u_{1}+u_{2}}{2}$. Substituting (1.15) in the numerator, we obtain asymptotics for $\mathbb{E}\left(\prod_{j=1}^{2}\left|P_{n}\left(u_{j}\right)\right|^{\alpha}\right)$.

This observation can be used to obtain information about extreme values of $\left|P_{n}(x)\right|$ for large $n$. This problem was investigated in [20], and in this context the authors needed large $n$ asymptotics for integrals of the form (see in particular [20, Section 2])

$$
I_{n}(\theta, \rho)=\int_{-\theta}^{\theta} \int_{-\theta}^{\theta} \mathbb{E}\left(\prod_{j=1}^{2}\left|P_{n}\left(u_{j}\right)\right|^{\alpha}\right) \prod_{j=1}^{2} e^{-\alpha n \lim _{k \rightarrow \infty}\left(\frac{1}{k} \mathbb{E} \log \left|P_{k}\left(u_{j}\right)\right|\right)} \rho\left(u_{j}\right) d u_{j},
$$

with $\theta \in[0,1], \rho$ strictly positive and continuous on $(-\theta, \theta)$, and $\alpha>0$. The authors note that one can differentiate (1.31) for $k=1$ with respect to $\alpha$ and evaluate at $\alpha=0$ to obtain

$$
\lim _{n \rightarrow \infty} \frac{1}{n} \mathbb{E}\left(\log \left|P_{n}(u)\right|\right)=u^{2}-1 / 2-\log 2,
$$

since $C(0)=1, C^{\prime}(0)=0$. In order to obtain asymptotics for $I_{n}$ as $n \rightarrow \infty$, one needs asymptotics for $\mathbb{E}\left(\prod_{j=1}^{2}\left|P_{n}\left(u_{j}\right)\right|^{\alpha}\right)$, both in the region where $u_{1}$ and $u_{2}$ are bounded away from each other (in this region, we can use Krasovsky's result (1.31)) and in the region where $u_{1}$ and $u_{2}$ are close to each other (in this region, we need to use our expansion (1.15)).

As a corollary to Theorem 1.1 we have

Corollary 1.11. Let $0<\theta<1, \alpha>0$, and let $\rho$ be a strictly positive continuous function on $(-\theta, \theta)$. Then,

$$
I_{n}(\theta, \rho)= \begin{cases}C_{1}(\alpha) n^{\alpha^{2} / 2}(1+o(1)) & \text { for } \alpha^{2}<2 \\ C_{2} n \log n(1+o(1)) & \text { for } \alpha^{2}=2 \\ C_{3}(\alpha) n^{\alpha^{2}-1}(1+o(1)) & \text { for } \alpha^{2}>2\end{cases}
$$

as $n \rightarrow \infty$, where

$$
\begin{aligned}
& C_{1}(\alpha)=\frac{G\left(1+\frac{\alpha}{2}\right)^{4}}{G(1+\alpha)^{2}} \int_{-\theta}^{\theta} \int_{-\theta}^{\theta} \frac{\left(\left(1-u_{1}^{2}\right)\left(1-u_{2}^{2}\right)\right)^{\alpha^{2} / 8}}{\left|u_{1}-u_{2}\right|^{\alpha^{2} / 2}} \rho\left(u_{1}\right) \rho\left(u_{2}\right) d u_{1} d u_{2}, \\
& C_{2}=2 \frac{G\left(1+\frac{1}{\sqrt{2}}\right)^{4}}{G(1+\sqrt{2})^{2}} \int_{-\theta}^{\theta}\left(1-u^{2}\right)^{1 / 2} \rho(u)^{2} d u \\
& C_{3}(\alpha)=2^{\alpha^{2}} \frac{G(\alpha+1)^{2}}{G(2 \alpha+1)} \int_{-\theta}^{\theta}\left(1-u^{2}\right)^{\frac{\alpha^{2}-1}{2}} \rho(u)^{2} d u \int_{0}^{\infty} \exp \left(\int_{0}^{-2 i v} \frac{\sigma_{\alpha}^{+}(s)-\alpha^{2}}{s} d s-i \alpha v\right) d v .
\end{aligned}
$$

Remark 1.12. For $\alpha^{2}<2$, the main contribution in the integral $I_{n}(\theta, \rho)$ comes from the asymptotics (1.31) in the region of integration where $u_{1}$ and $u_{2}$ are bounded away from each other. For $\alpha^{2}>2$, the main contribution comes from the asymptotics in Theorem 1.1 in the region of integration where $\left|u_{1}-u_{2}\right|=\mathcal{O}\left(n^{-1}\right)$.

Proof. First, we note that (1.31) can be extended: as $n \rightarrow \infty$ and $\left|u_{1}-u_{2}\right| \rightarrow 0$ sufficiently slowly such that $n\left|u_{1}-u_{2}\right| \rightarrow \infty$,

$$
\mathbb{E}\left(\prod_{j=1}^{2}\left|P_{n}\left(u_{j}\right)\right|^{\alpha}\right)=F\left(n, u_{1}, u_{2}, \frac{\alpha}{2}, \frac{\alpha}{2}\right)(1+o(1))
$$


with the error term uniform for $t_{0}>\left|u_{1}-u_{2}\right|>2 t_{n}$ for some $t_{0}>0$ and $t_{n} \rightarrow 0$ such that $n t_{n} \rightarrow \infty$. This follows by recalling the identity

$$
\lim _{s \rightarrow-i \infty}\left(\int_{0}^{s} \frac{\sigma_{\alpha}^{+}(\widetilde{s})-\alpha^{2}}{\widetilde{s}} d \widetilde{s}+\frac{\alpha s}{2}+\frac{\alpha^{2}}{2} \log |s|\right)=\log \frac{G\left(1+\frac{\alpha}{2}\right)^{4} G(1+2 \alpha)}{G(1+\alpha)^{4}},
$$

from [5, formula (1.26)] and applying it to (1.15), which one substitutes into (1.32).

We now split the integral in (1.33) in two parts: the integral over

$$
\mathcal{A}_{\theta, t_{n}}=\left\{\left(u_{1}, u_{2}\right):\left|u_{1}\right|,\left|u_{2}\right|<\theta,\left|u_{1}-u_{2}\right|>2 \sqrt{t_{n}}\right\},
$$

and the integral over $[-\theta, \theta]^{2} \backslash \mathcal{A}_{\theta, t_{n}}$, for some $t_{n}$ which converges sufficiently slowly to 0 as $n \rightarrow \infty$, slower than $n^{-2}$. To compute the contribution of the integral over $\mathcal{A}_{\theta, t_{n}}$, we can use (1.34), (1.31), and (1.35). For the contribution of the complement of $\mathcal{A}_{\theta, t_{n}}$, we substitute (1.15) in (1.32) and then, together with (1.34), in (1.33). Using (1.31) to compute the ratio $\widehat{Z}_{n}(0, \alpha, V) / \widehat{Z}_{n}^{\mathrm{GUE}}$ and changing variables $t=\left(u_{1}-u_{2}\right)^{2} / 4, u=\left(u_{1}+u_{2}\right) / 2$, we finally obtain

$$
\begin{aligned}
I_{n}(\theta, \rho)= & \left(\left(\frac{n}{2}\right)^{\alpha^{2} / 2} C(\alpha / 2)^{2} \iint_{\mathcal{A}_{\theta, t_{n}}} \frac{\left(\left(1-u_{1}^{2}\right)\left(1-u_{2}^{2}\right)\right)^{\alpha^{2} / 8}}{\left(2\left|u_{1}-u_{2}\right|\right)^{\alpha^{2} / 2}} \rho\left(u_{1}\right) \rho\left(u_{2}\right) d u_{1} d u_{2}\right. \\
& +2\left(\frac{n}{2}\right)^{\alpha^{2}} C(\alpha) \int_{-\theta}^{\theta}\left(1-u^{2}\right)^{\alpha^{2} / 2} \rho(u)^{2} \\
& \left.\times\left(\int_{0}^{t_{n}} \exp \left(\int_{0}^{\widehat{s}_{n, t}} \frac{\sigma_{\alpha}^{+}(s)-\alpha^{2}}{s} d s+\frac{\alpha \widehat{s}_{n, t}}{2}\right) \frac{d t}{\sqrt{t}}\right) d u\right)(1+o(1)),
\end{aligned}
$$

as $n \rightarrow \infty$, where

$$
\widehat{s}_{n, t}=-4 \pi i n \sqrt{t} \psi_{V}(0)=-8 i n \sqrt{t} \sqrt{1-u^{2}}
$$

in terms of the integration variables $t, u$. Here we need that the error terms found in Theorem 1.1 are uniform in $-\theta<u<\theta$ for the potential $V(x)=2(x+u)^{2}$. This is not mentioned elsewhere in this paper but can readily be seen to hold true by inspection of the proof of Theorem 1.1, if $|\theta|<1$. If $\theta=1$, contributions from the region where the singularities approach an edge point of the support of the equilibrium measure will have to be taken into account as well, but this is outside the scope of this paper.

From (1.36) and the small $s$ asymptotics of $\sigma_{\alpha}^{+}$it follows that with $v=4 n \sqrt{1-u^{2}} \sqrt{t}$,

$$
\begin{aligned}
\int_{0}^{t_{n}} & \exp \left(\int_{0}^{\widehat{s}_{n, t}} \frac{\sigma_{\alpha}^{+}(s)-\alpha^{2}}{s} d s+\frac{\alpha \widehat{s}_{n, t}}{2}\right) \frac{d t}{\sqrt{t}} \\
& =\frac{1}{2 n \sqrt{1-u^{2}}} \int_{0}^{4 n \sqrt{1-u^{2}} \sqrt{t_{n}}} \exp \left(\int_{0}^{-2 i v} \frac{\sigma_{\alpha}^{+}(s)-\alpha^{2}}{s} d s-i \alpha v\right) d v \\
& = \begin{cases}o\left(n^{-\alpha^{2} / 2}\right) & \text { for } \alpha^{2}<2, \\
\frac{1}{4 \sqrt{1-u^{2}}} \frac{G(1+\sqrt{2} / 2)^{4} G(1+2 \sqrt{2})}{G(1+\sqrt{2})^{4}} n^{-1} \log n+o\left(n^{-1} \log n\right) & \text { for } \alpha^{2}=2, \\
c n^{-1}+o\left(n^{-1}\right) & \text { for } \alpha^{2}>2,\end{cases}
\end{aligned}
$$

as $n \rightarrow \infty$, with

$$
c=\frac{1}{2 \sqrt{1-u^{2}}} \int_{0}^{\infty} \exp \left(\int_{0}^{-2 i v} \frac{\sigma_{\alpha}^{+}(s)-\alpha^{2}}{s} d s-i \alpha v\right) d v .
$$

Formulas (1.37) and (1.38) yield the corollary, where it is readily seen from (1.36) that the integral appearing in the constant $C_{3}$ is well defined. 


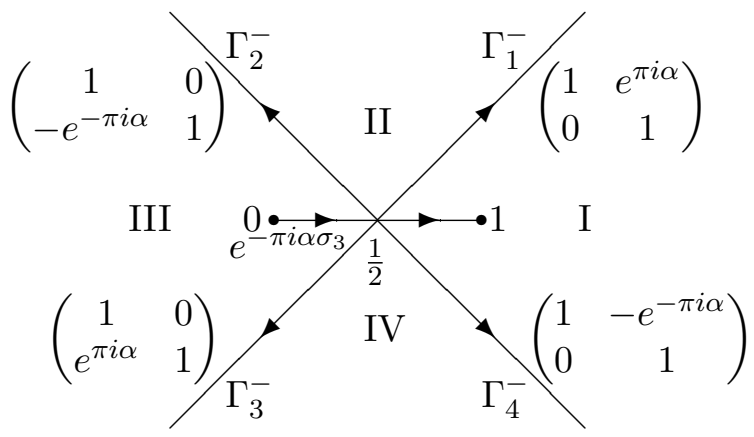

Figure 1. The jump contour $\Gamma^{-}$and the jump matrices for $\Psi^{-}$.

\subsection{Outline}

In Section 2, we recall a model RH problem, introduced in [4], associated to the fifth Painlevé equation. We define the functions $\Phi_{1}$ and $\Phi_{2}$ in terms of the solution to the RH problem for $\tau<0$, and describe their relation with the solution $\sigma_{\alpha}^{-}$to the fifth Painlevé equation. For $\alpha$ integer, we construct the $\mathrm{RH}$ solution recursively. In Section 3, we describe a second model $\mathrm{RH}$ problem which was introduced in [5], we define $\Phi_{1}$ and $\Phi_{2}$ in terms of its solution for $\tau>0$, and we relate them to the Painlevé solution $\sigma_{\alpha}^{+}$. For $\alpha$ even, we construct the $\mathrm{RH}$ solution recursively. In Section 4, we recall the standard RH problem which characterizes the orthogonal polynomials $p_{j}^{(n)}$, and we define the $g$-function needed to analyze this $\mathrm{RH}$ problem asymptotically. In Section 5, we perform a Deift/Zhou steepest descent analysis to obtain large $n$ asymptotics for the orthogonal polynomials in the case where $t<0$ (i.e., the case where the singularities are complex and approach 0 ). The construction of a local parametrix near 0 , in terms of the model problem defined in Section 2, is crucial here. At the end of Section 5, we will prove Theorems 1.7 and 1.1 in the case where $t<0$. In Section 6 , we perform a similar asymptotic analysis in the case $t>0$ (i.e., the case where the singularities are real and approach 0). Here the local parametrix is constructed in terms of the model problem from Section 3. This will allow us to prove Theorems 1.7 and 1.1 for $t>0$.

\section{Model RH problem for $\tau<0$}

The functions $\Phi_{1}$ and $\Phi_{2}$ that appeared in the limiting kernel $\mathbb{K}_{\alpha}^{\mathrm{PV}}$ can be defined in terms of a RH problem. We need to distinguish between the case where $\tau>0$ and $\tau<0$.

The first model RH problem is a special case of the one studied in [4, Section 1.3]. For our purposes, the parameter $\beta$ from [4] is set to zero. The RH problem depends on parameters $\alpha>-1 / 2$ and $s \in \mathbb{C}$. The relevant case for us will be $s>0$.

\section{RH problem for $\Psi^{-}$}

(a) $\Psi^{-}: \mathbb{C} \backslash \Gamma^{-} \rightarrow \mathbb{C}^{2 \times 2}$ is analytic, with $\Gamma^{-}=\Gamma_{1}^{-} \cup \Gamma_{2}^{-} \cup \Gamma_{3}^{-} \cup \Gamma_{4}^{-} \cup[0,1]$, and

$$
\Gamma_{1}^{-}=\frac{1}{2}+e^{\frac{\pi i}{4}} \mathbb{R}^{+}, \quad \Gamma_{2}^{-}=\frac{1}{2}+e^{\frac{3 \pi i}{4}} \mathbb{R}^{+}, \quad \Gamma_{3}^{-}=\frac{1}{2}+e^{-\frac{3 \pi i}{4}} \mathbb{R}^{+}, \quad \Gamma_{4}^{-}=\frac{1}{2}+e^{-\frac{\pi i}{4}} \mathbb{R}^{+},
$$

oriented as in Fig. 1.

(b) $\Psi^{-}$has continuous boundary values on $\Gamma^{-} \backslash\left\{0, \frac{1}{2}, 1\right\}$, which we denote by $\Psi_{+}^{-}(z)$ if the limit is taken from the left when oriented along the contour, and $\Psi_{-}^{-}(z)$ if the limit is taken from the right. We have the jump relations

$$
\Psi_{+}^{-}(z)=\Psi_{-}^{-}(z)\left(\begin{array}{cc}
1 & e^{\pi i \alpha} \\
0 & 1
\end{array}\right) \quad \text { for } z \in \Gamma_{1}^{-},
$$




$$
\begin{array}{ll}
\Psi_{+}^{-}(z)=\Psi_{-}^{-}(z)\left(\begin{array}{cc}
1 & 0 \\
-e^{-\pi i \alpha} & 1
\end{array}\right) & \text { for } z \in \Gamma_{2}^{-}, \\
\Psi_{+}^{-}(z)=\Psi_{-}^{-}(z)\left(\begin{array}{cc}
1 & 0 \\
e^{\pi i \alpha} & 1
\end{array}\right) & \text { for } z \in \Gamma_{3}^{-}, \\
\Psi_{+}^{-}(z)=\Psi_{-}^{-}(z)\left(\begin{array}{cc}
1 & -e^{-\pi i \alpha} \\
0 & 1
\end{array}\right) & \text { for } z \in \Gamma_{4}^{-}, \\
\Psi_{+}^{-}(z)=\Psi_{-}^{-}(z) e^{-\pi i \alpha \sigma_{3}} & \text { for } z \in(0,1),
\end{array}
$$

where $\sigma_{3}=\left(\begin{array}{cc}1 & 0 \\ 0 & -1\end{array}\right)$ is the third Pauli matrix.

(c) There exist $q, p, r$ independent of $z$ (but depending on $s, \alpha$ ) such that $\Psi^{-}(z)$ has the following behavior as $z \rightarrow \infty$ :

$$
\Psi^{-}(z)=\left(I+\frac{1}{z}\left(\begin{array}{cc}
q & r \\
p & -q
\end{array}\right)+\mathcal{O}\left(z^{-2}\right)\right) \exp \left(-\frac{s}{2} z \sigma_{3}\right) .
$$

(d) The functions $G(z):=\Psi^{-}(z) z^{-\frac{\alpha}{2} \sigma_{3}}$ and $H(z):=\Psi^{-}(z)(z-1)^{\frac{\alpha}{2} \sigma_{3}}$ are bounded for $z$ near 0 and 1 respectively, and $\Psi^{-}$is bounded near $1 / 2$. The branches in the definitions of $G, H$ are such that $z^{\frac{\alpha}{2}},(z-1)^{\frac{\alpha}{2}}>0$ on the + side of $(1, \infty)$.

For $\alpha>-1 / 2$, it was shown in [4, Theorem 1.8(i)] that the $\mathrm{RH}$ problem for $\Psi^{-}$has a unique solution for all $s>0$.

It will be useful to note that the following symmetry relation holds:

$$
e^{\frac{s}{2} \sigma_{3}} \Psi^{-}\left(z+\frac{1}{2}\right)=\sigma_{1} \Psi^{-}\left(-z+\frac{1}{2}\right) \sigma_{1}, \quad \sigma_{1}=\left(\begin{array}{cc}
0 & 1 \\
1 & 0
\end{array}\right) .
$$

It can indeed be verified that the left and right hand side satisfy the same, uniquely solvable, $\mathrm{RH}$ problem.

For $\tau<0$ and $u \in \mathbb{R}$, we define

$$
\left(\begin{array}{l}
\Phi_{1}(u ; \tau) \\
\Phi_{2}(u ; \tau)
\end{array}\right)=\Psi^{-}\left(z=-\frac{2 i u}{s}+\frac{1}{2} ; s\right) e^{\mp \pi i \alpha / 2 \sigma_{3}}\left(\begin{array}{c}
1 \\
-1
\end{array}\right) \quad \text { for } \pm u>0, \quad s=\sqrt{-\tau} .
$$

One verifies using the jump conditions for $\Psi^{-}$that $\Phi_{1}$ and $\Phi_{2}$ are analytic in a neighborhood of the real line. In fact, the singularities $z=0$ and $z=1$ of $\Psi^{-}$are transformed to complex conjugate singularities $u= \pm i s / 4= \pm i \sqrt{-\tau} / 4$ for $\Phi_{1}$ and $\Phi_{2}$. We have the asymptotic behavior

$$
\left(\begin{array}{l}
\Phi_{1}(u ; \tau) \\
\Phi_{2}(u ; \tau)
\end{array}\right)=\left(I+\mathcal{O}\left(u^{-1}\right)\right) \exp \left((i u-\sqrt{-\tau} / 4) \sigma_{3}\right) e^{\mp \pi i \alpha / 2 \sigma_{3}}\left(\begin{array}{c}
1 \\
-1
\end{array}\right)
$$

as $u \rightarrow \pm \infty$. The kernel $\mathbb{K}_{\alpha}^{\mathrm{PV}}(u, v ; \tau)$ is then given by (1.27) for $\tau<0$.

\subsection{Lax pair and Painlevé V}

There is a connecton between the $\mathrm{RH}$ problem for $\Psi^{-}$and the Painlevé V equation, which relies on the theory of isomonodromy preserving deformations [14]. We recall from [4] (in particular Theorem 1.8(ii) and Section 4.3 in that paper) that the $\mathrm{RH}$ solution $\Psi=\Psi^{-}$solves a system of linear differential equations

$$
\begin{aligned}
& \Psi_{z}(z ; s)=A(z ; s) \Psi(z ; s), \\
& \Psi_{s}(z ; s)=B(z ; s) \Psi(z ; s),
\end{aligned}
$$


where the matrices $A$ and $B$ have the form

$$
\begin{aligned}
& A(z ; s)=A_{\infty}(s)+\frac{A_{0}(s)}{z}+\frac{A_{1}(s)}{z-1}, \\
& B(z ; s)=B_{0}(s)+B_{1}(s) z .
\end{aligned}
$$

The $2 \times 2$ matrices $A_{0}, A_{1}, A_{\infty}, B_{0}, B_{1}$, which are independent of $z$ but can depend on $s$ and $\alpha$, can be parametrized as follows,

$$
\begin{aligned}
& A_{0}=\left(\begin{array}{cc}
-v+\alpha / 2 & u y(v-\alpha) \\
-\frac{v}{u y} & v-\alpha / 2
\end{array}\right), \\
& A_{1}=\left(\begin{array}{cc}
v-\alpha / 2 & -y(v-\alpha) \\
\frac{v}{y} & -v+\alpha / 2
\end{array}\right), \\
& A_{\infty}=-\frac{s}{2} \sigma_{3}, \quad B_{1}=-\frac{1}{2} \sigma_{3}, \quad B_{0}=\frac{A_{0}+A_{1}}{s} .
\end{aligned}
$$

Here $u, v$, and $y$ are functions of $s$ and $\alpha$. We have the following relation between $u, v$ and the functions $q, r, p$ appearing in the asymptotic expansion (2.1) for $\Psi^{-}$as $z \rightarrow \infty$,

$$
\begin{aligned}
& v=\frac{\alpha}{2}-q-s r p, \\
& u=1+\frac{s p}{(1-s) p+s p^{\prime}} .
\end{aligned}
$$

The compatibility condition $\Psi_{s z}=\Psi_{z s}$ implies that $A_{s}-B_{z}+A B-B A=0$, and this implies the following system of equations for $u, v, y$ :

$$
\begin{aligned}
& s u_{s}=s u+(\alpha-2 v)(u-1)^{2}, \\
& s v_{s}=v\left(u-\frac{1}{u}\right)(v-\alpha), \\
& s y_{s}=y\left(-2 v+\alpha+u v-u \alpha+\frac{v}{u}-s\right) .
\end{aligned}
$$

By eliminating $v$ from the top two equations, we obtain a special case of the fifth Painlevé equation,

$$
u_{s s}=\left(\frac{1}{2 u}+\frac{1}{u-1}\right) u_{s}^{2}-\frac{1}{s} u_{s}+\frac{(1-u)^{2}}{s^{2}}\left(\frac{\alpha^{2}}{2}\left(u-\frac{1}{u}\right)\right)+\frac{u}{s}-\frac{u(u+1)}{2(u-1)} .
$$

If we define $\sigma(s)$ as

$$
\sigma(s)=\int_{s}^{+\infty} v(\xi) d \xi
$$

(it was shown in [4] that $v$ decays rapidly as $s \rightarrow+\infty$ so that this integral converges), then $\sigma$ solves (1.10).

\subsection{Recursive construction of $\Psi^{-}$if $\alpha \in \mathbb{N}$}

For positive integer values of $\alpha$, the $\mathrm{RH}$ solution $\Psi^{-}$can be constructed explicitly. We will denote $\Psi_{\alpha}^{-}$instead of $\Psi^{-}$in this section to emphasize the dependence on $\alpha$. The crucial observation here is that the jump matrices for $\Psi_{\alpha}^{-}$are periodic in $\alpha$ : they remain the same if we replace $\alpha$ by $\alpha+2$. This fact indicates that there is a Schlesinger type transformation [28] relating $\Psi_{\alpha}$ to $\Psi_{\alpha+2}$. Even more, if we replace $\alpha$ by $\alpha+1$, the jump matrices are modified in 
a simple way: the jumps on the diagonals $\Gamma_{1}^{-}, \Gamma_{2}^{-}, \Gamma_{3}^{-}, \Gamma_{4}^{-}$are the same for the functions $\Psi_{\alpha}^{-}$ and $\sigma_{3} \Psi_{\alpha+1}^{-} \sigma_{3}$, whereas they are opposite on $(0,1)$. Based on this observation, and on the fact that the solution for $\alpha=0$ is simple and explicit, we can recursively construct a solution for any integer $\alpha$.

For positive integer $\alpha$ and $s \in(0,+\infty)$, define $X_{\alpha}$ as follows,

$$
X_{\alpha}(z ; s)= \begin{cases}\Psi_{\alpha}^{-}(z ; s) & \text { for } z \in \mathrm{II}, \mathrm{IV}, \\
\Psi_{\alpha}^{-}(z ; s)\left(\begin{array}{cc}
1 & (-1)^{\alpha} \\
0 & 1
\end{array}\right) & \text { for } z \in \mathrm{I}, \\
\Psi_{\alpha}^{-}(z ; s)\left(\begin{array}{cc}
1 & 0 \\
(-1)^{\alpha} & 1
\end{array}\right) & \text { for } z \in \mathrm{III},\end{cases}
$$

with regions I, II, III, IV as in Fig. 1. Then, by the RH conditions for $\Psi_{\alpha}^{-}, X_{\alpha}$ satisfies the following $\mathrm{RH}$ problem.

\section{RH problem for $X_{\alpha}$}

(a) $X_{\alpha}: \mathbb{C} \backslash[0,1] \rightarrow \mathbb{C}^{2 \times 2}$ is analytic.

(b) For $z \in(0,1)$, we have the jump relation $X_{\alpha,+}(z)=(-1)^{\alpha} X_{\alpha,-}(z)$.

(c) As $z \rightarrow \infty$,

$$
X_{\alpha}(z)=\left(I+\mathcal{O}\left(z^{-1}\right)\right) \exp \left(-\frac{s z}{2} \sigma_{3}\right)
$$

(d) The functions $G_{\alpha}$ and $H_{\alpha}$ defined by

$$
\begin{aligned}
& G_{\alpha}(z)=X_{\alpha}(z)\left(\begin{array}{cc}
1 & 0 \\
-(-1)^{\alpha} & 1
\end{array}\right) z^{-\frac{\alpha}{2} \sigma_{3}}, \\
& H_{\alpha}(z)=X_{\alpha}(z)\left(\begin{array}{cc}
1 & -(-1)^{\alpha} \\
0 & 1
\end{array}\right)(z-1)^{\frac{\alpha}{2} \sigma_{3}},
\end{aligned}
$$

are analytic at $z=0$ and at $z=1$ respectively. The branches in the definitions of $G_{\alpha}, H_{\alpha}$ are such that $z^{\frac{\alpha}{2}},(z-1)^{\frac{\alpha}{2}}>0$ on the + side of $(1, \infty)$.

For $\alpha=0$, this $\mathrm{RH}$ problem is easy to solve: we have

$$
X_{0}(z)=\exp \left(-\frac{s z}{2} \sigma_{3}\right) .
$$

Define

$$
W_{\alpha}(z)=\left(\frac{z}{z-1}\right)^{\frac{1}{2} \sigma_{3}} X_{\alpha}(z) \sigma_{3} X_{\alpha+1}(z)^{-1} \sigma_{3},
$$

where the square root is analytic on $\mathbb{C} \backslash[0,1]$ and positive for large positive $z$. We will explicitly construct $W_{\alpha}$ in terms of $G_{\alpha}$ and $H_{\alpha}$, and thus we will have the recursive relation

$$
X_{\alpha+1}(z)=\sigma_{3} W_{\alpha}(z)^{-1}\left(\frac{z}{z-1}\right)^{\frac{1}{2} \sigma_{3}} X_{\alpha}(z) \sigma_{3} .
$$

From the jump relations for $X_{\alpha}$, one verifies that $W_{\alpha}$ is meromorphic in $z$, with singularities at 0 and 1 , and as $z \rightarrow \infty$, we have

$$
W_{\alpha}(z)=I+\mathcal{O}\left(\frac{1}{z}\right)
$$


By condition (d) of the $\mathrm{RH}$ problem for $X_{\alpha}$, we obtain

$$
\begin{array}{ll}
W_{\alpha}(z)=\left(\frac{z}{z-1}\right)^{\frac{1}{2} \sigma_{3}} G_{\alpha}(z) z^{-\frac{1}{2} \sigma_{3}} \sigma_{3} G_{\alpha+1}(z)^{-1} \sigma_{3} & \text { for } z \text { near } 0 \\
W_{\alpha}(z)=\left(\frac{z}{z-1}\right)^{\frac{1}{2} \sigma_{3}} H_{\alpha}(z)(z-1)^{\frac{1}{2} \sigma_{3}} \sigma_{3} H_{\alpha+1}(z)^{-1} \sigma_{3} & \text { for } z \text { near } 1 .
\end{array}
$$

It follows from (2.24)-(2.26) that $W_{\alpha}$ takes the form

$$
W_{\alpha}(z)=I+\frac{P_{\alpha}}{z}+\frac{Q_{\alpha}}{z-1},
$$

for matrices $P_{\alpha}, Q_{\alpha}$ which are independent of $z$. Moreover, it is easily verified that $X_{\alpha}$ exhibits the following symmetry,

$$
X_{\alpha}\left(z+\frac{1}{2}\right)=e^{-\frac{s}{2} \sigma_{3}} \sigma_{1} X_{\alpha}\left(-z+\frac{1}{2}\right) \sigma_{1}
$$

which yields the relation

$$
-e^{-\frac{s}{2} \sigma_{3}} \sigma_{1} P_{\alpha} \sigma_{1} e^{\frac{s}{2} \sigma_{3}}=Q_{\alpha}
$$

Condition (2.25) gives us that

$$
z^{\frac{1}{2} \sigma_{3}} G_{\alpha}(z)^{-1} z^{-\frac{1}{2} \sigma_{3}} \sigma_{3} W_{\alpha}(z) \text { is analytic in a neighborhood of } 0 .
$$

By substituting (2.27) into (2.30) it follows that the first row of $P_{\alpha}$ is 0 , and consequently, by $(2.30)$,

$$
z^{\frac{1}{2} \sigma_{3}} G_{\alpha}(0)^{-1} z^{-\frac{1}{2} \sigma_{3}} \sigma_{3} W_{\alpha}(z) \text { is analytic in a neighborhood of } 0 .
$$

This implies

$$
\begin{aligned}
& G_{\alpha, 21}(0)\left(1+P_{\alpha, 22}\right)+G_{\alpha, 11}(0) P_{\alpha, 21}=0 \\
& e^{-s} G_{\alpha, 21}(0) P_{\alpha, 21}+G_{\alpha, 11}(0) P_{\alpha, 22}=0 .
\end{aligned}
$$

By the unique solvability of the RH problem for $X_{\alpha}$, it follows that this linear system has a unique solution for $P_{\alpha, 22}$ and $P_{\alpha, 21}$ for any positive $s$.

In conclusion, since we know $X_{0}(z)$, we can compute $G_{0}(0)$ by $(2.20)$, and from that we can compute $P_{0}$ by (2.32), (2.33). Substituting those in (2.29), (2.27), and (2.23), we obtain $X_{1}(z)$. Similarly, once we know $X_{1}(z)$, we can compute $G_{1}(0), P_{1}$, and $X_{2}$, and so on.

For $\alpha=1$ and $\alpha=2$, in this way, we obtain the expressions

$$
\begin{aligned}
& X_{1}(z)=\frac{1}{\left(e^{s}-1\right) \sqrt{(z-1) z}}\left(\begin{array}{cc}
1+\left(e^{s}-1\right) z & -1 \\
e^{s} & -e^{s}+z\left(e^{s}-1\right)
\end{array}\right) e^{-\frac{s z}{2} \sigma_{3}}, \\
& X_{2}(z)=\frac{1}{\left(1-e^{s}\left(2+s^{2}\right)+e^{2 s}\right)(z-1) z} \\
& \times\left(\begin{array}{c}
1+e^{s}(s-1)-2 z+e^{s} z\left(2-2 s+s^{2}\right)+z^{2}-e^{s} z^{2}\left(2+s^{2}-e^{s}\right) \\
e^{s}\left(-1-s+e^{s}+z\left(2+s+e^{s}(-2+s)\right)\right)
\end{array}\right. \\
& \left.\begin{array}{c}
1-e^{s}+e^{s} s-z\left(2+s+e^{s}(s-2)\right) \\
e^{s}\left(-1-s+e^{s}\right)+z e^{s}\left(2+2 s+s^{2}-2 e^{s}\right)+z^{2}+z^{2} e^{s}\left(-2-s^{2}+e^{s}\right)
\end{array}\right) e^{-\frac{s z}{2} \sigma_{3}} .
\end{aligned}
$$


Expanding $X_{\alpha}$ as $z \rightarrow \infty$, we can obtain expressions for the Painlevé V functions $q(s), r(s)$, $p(s)$ by (2.1). In particular, for $\alpha=0,1,2$, we obtain

$$
q_{0}(s)=0, \quad q_{1}(s)=\frac{1}{e^{s}-1}+\frac{1}{2}, \quad q_{2}(s)=\frac{-1+e^{s}\left(-2 s+e^{s}\right)}{1-e^{s}\left(2+s^{2}\right)+e^{2 s}} .
$$

Using the relation (see [4, equation (4.109)])

$$
\sigma_{\alpha}^{-}(s)=s q(s)-\frac{\alpha s}{2},
$$

we can compute $\sigma_{0}^{-}, \sigma_{1}^{-}$, and $\sigma_{2}^{-}$: this gives (1.12)-(1.14).

\subsection{The kernel $\mathbb{K}_{\alpha}^{\mathrm{PV}}$ as $\tau \rightarrow-\infty$}

We follow the steps of [4, Section 4.1] in the analysis as $\tau \rightarrow-\infty$. Define

$$
\widetilde{\Psi}(z)=\Psi^{-}(z)\left(\frac{z-1}{z}\right)^{\frac{\alpha}{2} \sigma_{3}},
$$

where the power $\alpha / 2$ is taken analytic except on $[0,1]$ and tending to 1 as $z \rightarrow+\infty$. The fraction cancels out the jump of $\Psi^{-}$on $(0,1)$ and the singularities at 0 and 1 , and $\widetilde{\Psi}$ solves the following $\mathrm{RH}$ problem.

\section{RH problem for $\widetilde{\Psi}$}

(a) $\widetilde{\Psi}: \mathbb{C} \backslash\left(\Gamma_{1}^{-} \cup \Gamma_{2}^{-} \cup \Gamma_{3}^{-} \cup \Gamma_{4}^{-}\right)$.

(b) $\widetilde{\Psi}$ has the jump relations

$$
\begin{array}{ll}
\widetilde{\Psi}_{+}(z)=\widetilde{\Psi}_{-}(z)\left(\begin{array}{cc}
1 & e^{\pi i \alpha}\left(\frac{z-1}{z}\right)^{-\alpha} \\
0 & 1
\end{array}\right) & \text { for } z \in \Gamma_{1}^{-}, \\
\widetilde{\Psi}_{+}(z)=\widetilde{\Psi}_{-}(z)\left(\begin{array}{cc}
1 & 0 \\
-e^{-\pi i \alpha}\left(\frac{z-1}{z}\right)^{\alpha} & 1
\end{array}\right) & \text { for } z \in \Gamma_{2}^{-}, \\
\widetilde{\Psi}_{+}(z)=\widetilde{\Psi}_{-}(z)\left(\begin{array}{cc}
1 & 0 \\
e^{\pi i \alpha}\left(\frac{z-1}{z}\right)^{\alpha} & 1
\end{array}\right) & \text { for } z \in \Gamma_{3}^{-}, \\
\widetilde{\Psi}_{+}(z)=\widetilde{\Psi}_{-}(z)\left(\begin{array}{cc}
1 & -e^{-\pi i \alpha}\left(\frac{z-1}{z}\right)^{-\alpha} \\
0 & 1
\end{array}\right) & \text { for } z \in \Gamma_{4}^{-} .
\end{array}
$$

(c) As $z \rightarrow \infty$,

$$
\widetilde{\Psi}(z)=\left(I+\mathcal{O}\left(z^{-1}\right)\right) \exp \left(-\frac{s}{2} z \sigma_{3}\right) .
$$

Now we will deform the jump contour in such a way that it stays away from 0,1 , and $1 / 2$, to a contour as shown in Fig. 2. Define

$$
\widehat{\Psi}(z)= \begin{cases}e^{\frac{s}{4} \sigma_{3}} \widetilde{\Psi}(z) \exp \left(\frac{s}{2}\left(z-\frac{1}{2}\right) \sigma_{3}\right) & \text { for } z \notin \mathcal{A}_{1} \cup \mathcal{A}_{2}, \\
e^{\frac{s}{4} \sigma_{3}} \widetilde{\Psi}(z)\left(\begin{array}{cc}
1 & 0 \\
\left(\frac{1-z}{z}\right)^{\alpha} & 1
\end{array}\right) \exp \left(\frac{s}{2}\left(z-\frac{1}{2}\right) \sigma_{3}\right) & \text { for } z \in \mathcal{A}_{1}, \\
e^{\frac{s}{4} \sigma_{3}} \widetilde{\Psi}(z)\left(\begin{array}{cc}
1 & \left(\frac{1-z}{z}\right)^{-\alpha} \\
0 & 1
\end{array}\right) \exp \left(\frac{s}{2}\left(z-\frac{1}{2}\right) \sigma_{3}\right) & \text { for } z \in \mathcal{A}_{2},\end{cases}
$$

with $\mathcal{A}_{1}$ and $\mathcal{A}_{2}$ given in Fig. 2. Here, $\left(\frac{1-z}{z}\right)^{ \pm \alpha}$ is analytic except on $(-\infty, 0] \cup[1,+\infty)$. Then $\widehat{\Psi}$ satisfies a small norm RH problem as $s \rightarrow+\infty$. 


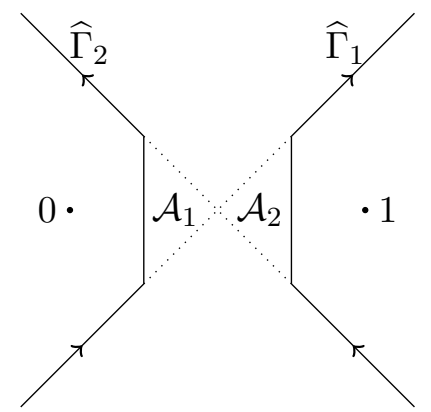

Figure 2. Contour $\widehat{\Gamma}$.

\section{RH problem for $\widehat{\Psi}$}

(a) $\widehat{\Psi}: \mathbb{C} \backslash \widehat{\Gamma} \rightarrow \mathbb{C}^{2 \times 2}$ is analytic, with $\widehat{\Gamma}=\widehat{\Gamma}_{1} \cup \widehat{\Gamma}_{2}$ given in Fig. 2 .

(b) $\widehat{\Psi}$ has the following jumps on $\widehat{\Gamma}$ :

$$
\begin{array}{ll}
\widehat{\Psi}_{+}(z)=\widehat{\Psi}_{-}(z)\left(\begin{array}{ccc}
1 & \left(\frac{1-z}{z}\right)^{-\alpha} e^{-s\left(z-\frac{1}{2}\right)} \\
0 & 1
\end{array}\right) & \text { for } z \in \widehat{\Gamma}_{1}, \\
\widehat{\Psi}_{+}(z)=\widehat{\Psi}_{-}(z)\left(\begin{array}{cc}
1 & 0 \\
-\left(\frac{1-z}{z}\right)^{\alpha} e^{s\left(z-\frac{1}{2}\right)} & 1
\end{array}\right) & \text { for } z \in \widehat{\Gamma}_{2} .
\end{array}
$$

As $s \rightarrow+\infty$, this means that

$$
\widehat{\Psi}_{+}(z)=\widehat{\Psi}_{-}(z)(I+\mathcal{O}(\exp (-c s))
$$

on $\widehat{\Gamma}_{1}$ and $\widehat{\Gamma}_{2}$, for some constant $c>0$.

(c) $\widehat{\Psi}(z)=I+\mathcal{O}\left(z^{-1}\right)$ as $z \rightarrow \infty$.

By standard small norm analysis, we have

$$
\widehat{\Psi}(z)=I+\mathcal{O}\left(\frac{1}{|z|+1} e^{-c s}\right), \quad s \rightarrow+\infty
$$

uniformly for $z \in \mathbb{C} \backslash \widehat{\Gamma}$. Inverting the transformations $\Psi^{-} \mapsto \widetilde{\Psi} \mapsto \widehat{\Psi}$ and using (2.3), we obtain

$$
\left(\begin{array}{l}
\Phi_{1}\left(x ;-s^{2}\right) \\
\Phi_{2}\left(x ;-s^{2}\right)
\end{array}\right)=\widehat{\Psi}\left(\frac{-2 i x}{s}+\frac{1}{2} ; s\right)\left(\frac{-4 i x-s}{-4 i x+s}\right)^{-\frac{\alpha}{2} \sigma_{3}} e^{\mp \frac{\pi i \alpha}{2} \sigma_{3}} e^{i x \sigma_{3}}\left(\begin{array}{c}
1 \\
-1
\end{array}\right), \quad \pm x>0 .
$$

By $(2.41)$, as $s \rightarrow+\infty, x \in \mathbb{R}$,

$$
\left(\begin{array}{l}
\Phi_{1}\left(\pi u ;-s^{2}\right) \\
\Phi_{2}\left(\pi u ;-s^{2}\right)
\end{array}\right)=\left(\begin{array}{c}
e^{\pi i u} \\
-e^{-\pi i u}
\end{array}\right)+\mathcal{O}\left(s^{-1}\right)
$$

which, by (1.27), yields the sine kernel limit as $s \rightarrow+\infty$,

$$
\mathbb{K}_{\alpha}^{\mathrm{PV}}\left(u, v ;-s^{2}\right)=\frac{\sin \pi(u-v)}{\pi(u-v)}+\mathcal{O}\left(s^{-1}\right),
$$

and (1.28) is proved. In fact, it is clear from this derivation that (1.28) holds also as $u, v$ tend to infinity together with $s$, as long as $u-v$ converges. 


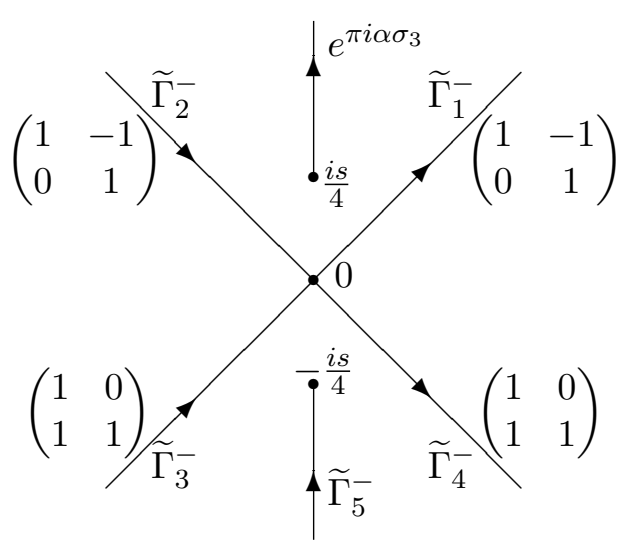

Figure 3. The jump contour $\widetilde{\Gamma}^{-}$and the jump matrices for $\Psi^{(1)}$.

\subsection{The kernel $\mathbb{K}_{\alpha}^{\mathrm{PV}}$ as $\tau \rightarrow 0$}

We will now study the RH problem for $\Psi^{-}$in the limit where $s \rightarrow 0$ and prove (1.29).

Define

$$
\Psi^{(1)}(z ; s)=e^{\frac{s}{4} \sigma_{3}} \Psi^{-}\left(-\frac{2 i z}{s}+\frac{1}{2} ; s\right) \chi(z),
$$

where

$$
\chi(z)= \begin{cases}e^{-\frac{\pi i \alpha}{2} \sigma_{3}} & \text { for } \operatorname{Re} z>0 \\ e^{\frac{\pi i \alpha}{2} \sigma_{3}} & \text { for } \operatorname{Re} z<0 .\end{cases}
$$

Then $\Psi^{(1)}$ satisfies the following $\mathrm{RH}$ problem.

\section{RH problem for $\Psi^{(1)}$}

(a) $\Psi^{(1)}: \mathbb{C} \backslash \widetilde{\Gamma}^{-} \rightarrow \mathbb{C}^{2 \times 2}$ is analytic, where

$$
\begin{array}{ll}
\widetilde{\Gamma}=\bigcup_{i=1}^{5} \widetilde{\Gamma}_{i}^{-}, & \widetilde{\Gamma}_{1}^{-}=\{z: \arg z=\pi / 4\}, \\
\widetilde{\Gamma}_{2}^{-}=\{z: \arg z=3 \pi / 4\}, & \widetilde{\Gamma}_{3}^{-}=\{z: \arg z=5 \pi / 4\}, \\
\widetilde{\Gamma}_{4}^{-}=\{z: \arg z=7 \pi / 4\}, & \widetilde{\Gamma}_{5}^{-}=i \mathbb{R} \backslash[-i s / 4, i s / 4] .
\end{array}
$$

The orientation is as in Fig. 3. Note that the orientation is different compared to the one in the jump contour for $\Psi^{-}$.

(b) $\Psi^{(1)}$ has continuous boundary values on $\widetilde{\Gamma}^{-} \backslash\{0\}$ :

$$
\begin{array}{ll}
\Psi_{+}^{(1)}(z)=\Psi_{-}^{(1)}(z)\left(\begin{array}{cc}
1 & -1 \\
0 & 1
\end{array}\right) & \text { for } z \in \widetilde{\Gamma}_{1}^{-} \cup \widetilde{\Gamma}_{2}^{-}, \\
\Psi_{+}^{(1)}(z)=\Psi_{-}^{(1)}(z)\left(\begin{array}{cc}
1 & 0 \\
1 & 1
\end{array}\right) & \text { for } z \in \widetilde{\Gamma}_{3}^{-} \cup \widetilde{\Gamma}_{4}^{-}, \\
\Psi_{+}^{(1)}(z)=\Psi_{-}^{(1)}(z) e^{\pi i \alpha \sigma_{3}} & \text { for } z \in \widetilde{\Gamma}_{5}^{-} .
\end{array}
$$

(c) $\Psi^{(1)}$ has the following behavior as $z \rightarrow \infty$ :

$$
\Psi^{(1)}(z)=\left(I+\mathcal{O}\left(z^{-1}\right)\right) e^{i z \sigma_{3}} \chi(z)
$$




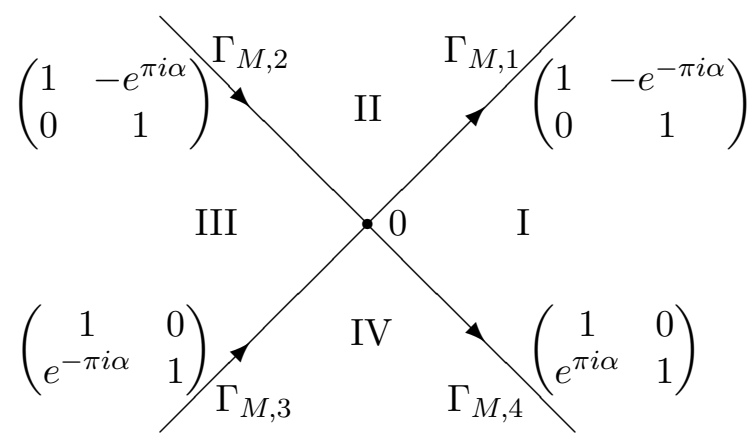

Figure 4. The contour $\Gamma_{M}$ and the jump matrices for $M$.

(d0) $\Psi^{(1)}$ has the following behavior as $z \rightarrow-i s / 4$ :

$$
\Psi^{(1)}(z)=\left(\begin{array}{ll}
\mathcal{O}\left(\left|z+\frac{i s}{4}\right|^{\alpha / 2}\right) & \mathcal{O}\left(\left|z+\frac{i s}{4}\right|^{-\alpha / 2}\right) \\
\mathcal{O}\left(\left|z+\frac{i s}{4}\right|^{\alpha / 2}\right) & \mathcal{O}\left(\left|z+\frac{i s}{4}\right|^{-\alpha / 2}\right)
\end{array}\right)
$$

(d1) $\Psi^{(1)}$ has the following behavior as $z \rightarrow i s / 4$ :

$$
\Psi^{(1)}(z)=\left(\begin{array}{ll}
\mathcal{O}\left(\left|z-\frac{i s}{4}\right|^{-\alpha / 2}\right) & \mathcal{O}\left(\left|z-\frac{i s}{4}\right|^{\alpha / 2}\right) \\
\mathcal{O}\left(\left|z-\frac{i s}{4}\right|^{-\alpha / 2}\right) & \mathcal{O}\left(\left|z-\frac{i s}{4}\right|^{\alpha / 2}\right)
\end{array}\right)
$$

(e) $\Psi^{(1)}$ is bounded at 0 .

Using (2.3) and (2.42), we obtain

$$
\left(\begin{array}{c}
\Phi_{1}\left(x ;-s^{2}\right) \\
\Phi_{2}\left(x ;-s^{2}\right)
\end{array}\right)=e^{-\frac{s}{4} \sigma_{3}} \Psi^{(1)}(x ; s)\left(\begin{array}{c}
1 \\
-1
\end{array}\right) .
$$

To study $\Psi^{(1)}(z ; s)$ as $s \rightarrow 0$, we need to recall results from [4, Sections 4.2 .1 and 4.2.2]. The asymptotics as $s \rightarrow 0$ can be described as follows: we have for $|z|>\delta$ with $\delta>0$ arbitrary that

$$
\Psi^{(1)}(z ; s)=\left(I+\mathcal{O}(|s \log | s||)+\mathcal{O}\left(s^{1+2 \alpha}\right)\right) M(z) e^{\mp \frac{\pi i \alpha}{2} \sigma_{3}} \quad \text { for } \pm \operatorname{Re} z>0 .
$$

Here $M$ satisfies the following $\mathrm{RH}$ conditions.

\section{RH problem for $M$}

(a) $M: \mathbb{C} \backslash \Gamma_{M} \rightarrow \mathbb{C}^{2 \times 2}$ is analytic, where

$$
\begin{array}{ll}
\Gamma_{M}=\cup_{i=1}^{5} \Gamma_{M, i}, & \\
\Gamma_{M, 1}=\{z: \arg (z)=\pi / 4\}, & \Gamma_{M, 2}=\{z: \arg (z)=3 \pi / 4\}, \\
\Gamma_{M, 3}=\{z: \arg (z)=5 \pi / 4\}, & \Gamma_{M, 4}=\{z: \arg (z)=7 \pi / 4\} .
\end{array}
$$

The orientation is as in Fig. 4.

(b) $M$ has continuous boundary values on $\Gamma_{M} \backslash\{0\}$ :

$$
\begin{array}{ll}
M_{+}(z)=M_{-}(z)\left(\begin{array}{cc}
1 & -e^{-\pi i \alpha} \\
0 & 1
\end{array}\right) & \text { for } z \in \Gamma_{M, 1}, \\
M_{+}(z)=M_{-}(z)\left(\begin{array}{cc}
1 & -e^{\pi i \alpha} \\
0 & 1
\end{array}\right) & \text { for } z \in \Gamma_{M, 2},
\end{array}
$$




$$
\begin{array}{ll}
M_{+}(z)=M_{-}(z)\left(\begin{array}{cc}
1 & 0 \\
e^{-\pi i \alpha} & 1
\end{array}\right) & \text { for } z \in \Gamma_{M, 3}, \\
M_{+}(z)=M_{-}(z)\left(\begin{array}{cc}
1 & 0 \\
e^{\pi i \alpha} & 1
\end{array}\right) & \text { for } z \in \Gamma_{M, 4} .
\end{array}
$$

(c) $M$ has the following behavior as $z \rightarrow \infty$ :

$$
M(z)=\left(I+\mathcal{O}\left(z^{-1}\right)\right) e^{i z \sigma_{3}}
$$

The RH problem for $M$ has an explicit solution (which is not unique, unless one adds a condition to control the behaviour of $M$ near 0) given in terms of confluent hypergeometric functions which is used in $[4,5]$. But in the special case we are dealing with, the confluent hypergeometric functions degenerate to Hankel and modified Bessel functions $H_{\alpha}^{(1)}, H_{\alpha}^{(2)}, I_{\alpha}$ and $K_{\alpha}$ (see [29]), and we use an explicit formula for $M$ similar to the one given in [27, 31]. Writing

$$
K=\frac{1}{\sqrt{2}}\left(\begin{array}{cc}
-1 & 1 \\
-1 & -1
\end{array}\right) e^{-\frac{\pi i}{4} \sigma_{3}}
$$

we have

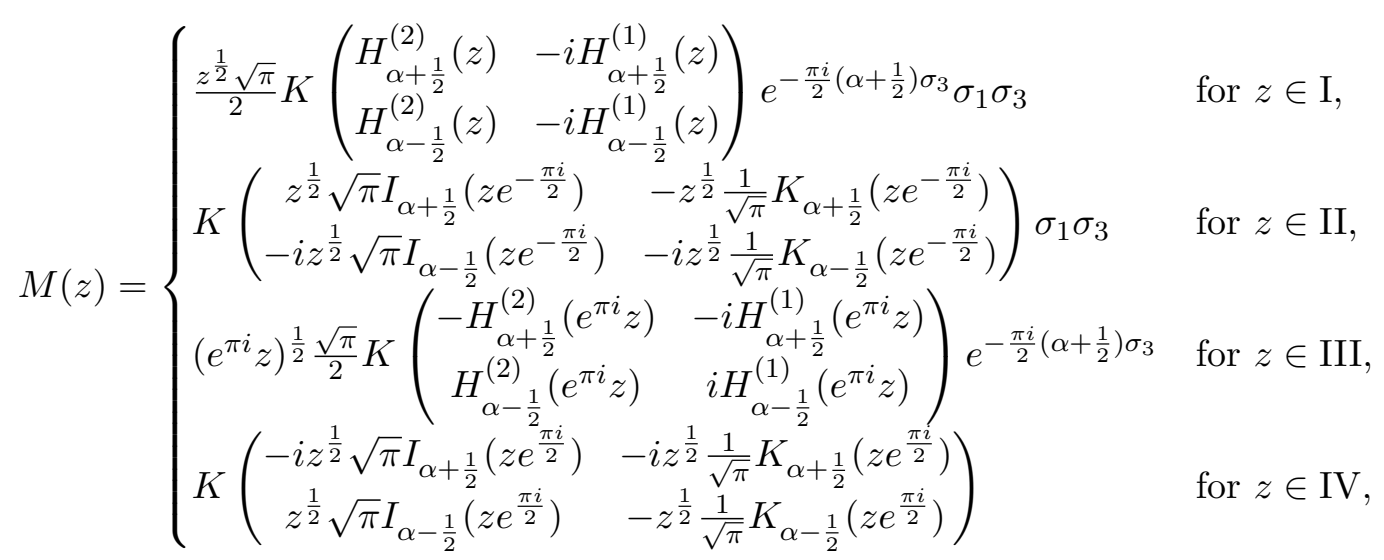

where the square roots and the Hankel and Bessel functions have branch cuts on $(-\infty, 0]$.

By (2.46) and (2.45), we have, for $\pm x>0$,

$$
\left(\begin{array}{l}
\Phi_{1}\left(x ;-s^{2}\right) \\
\Phi_{2}\left(x ;-s^{2}\right)
\end{array}\right)=M(x ; s) e^{\mp \frac{\pi i \alpha}{2} \sigma_{3}}\left(\begin{array}{c}
1 \\
-1
\end{array}\right)+\mathcal{O}(|s| \log |s|)+\mathcal{O}\left(|s|^{1+2 \alpha}\right), \quad s \rightarrow 0 .
$$

Using (1.27) and substituting in (2.47), one obtains that as $\tau \rightarrow 0$ :

$$
\begin{aligned}
& \mathbb{K}_{\alpha}^{\mathrm{PV}}(u, v ; \tau)=\frac{\pi \sqrt{u v}}{8(u-v)}\left(\left(H_{\alpha-\frac{1}{2}}^{(1)}(\pi v)+H_{\alpha-\frac{1}{2}}^{(2)}(\pi v)\right)\left(H_{\alpha+\frac{1}{2}}^{(1)}(\pi u)+H_{\alpha+\frac{1}{2}}^{(2)}(\pi u)\right)\right. \\
& \left.-\left(H_{\alpha+\frac{1}{2}}^{(1)}(\pi v)+H_{\alpha+\frac{1}{2}}^{(2)}(\pi v)\right)\left(H_{\alpha-\frac{1}{2}}^{(1)}(\pi u)+H_{\alpha-\frac{1}{2}}^{(2)}(\pi u)\right)\right)+\mathcal{O}\left(|\tau|^{1 / 2} \log |\tau|\right)+\mathcal{O}\left(|\tau|^{\frac{1}{2}+\alpha}\right) .
\end{aligned}
$$

The identity $H_{\alpha}^{(1)}(z)+H_{\alpha}^{(2)}(z)=2 J_{\alpha}(z)$ yields our result: as $\tau \rightarrow 0$ we have

$$
\begin{aligned}
\mathbb{K}_{\alpha}^{\mathrm{PV}}(u, v ; \tau)= & \frac{\pi \sqrt{u v}}{2(u-v)}\left(J_{\alpha-\frac{1}{2}}(\pi v) J_{\alpha+\frac{1}{2}}(\pi u)-J_{\alpha+\frac{1}{2}}(\pi v) J_{\alpha-\frac{1}{2}}(\pi u)\right) \\
& +\mathcal{O}\left(|\tau|^{1 / 2} \log |\tau|\right)+\mathcal{O}\left(|\tau|^{\frac{1}{2}+\alpha}\right) .
\end{aligned}
$$

This proves (1.29). 


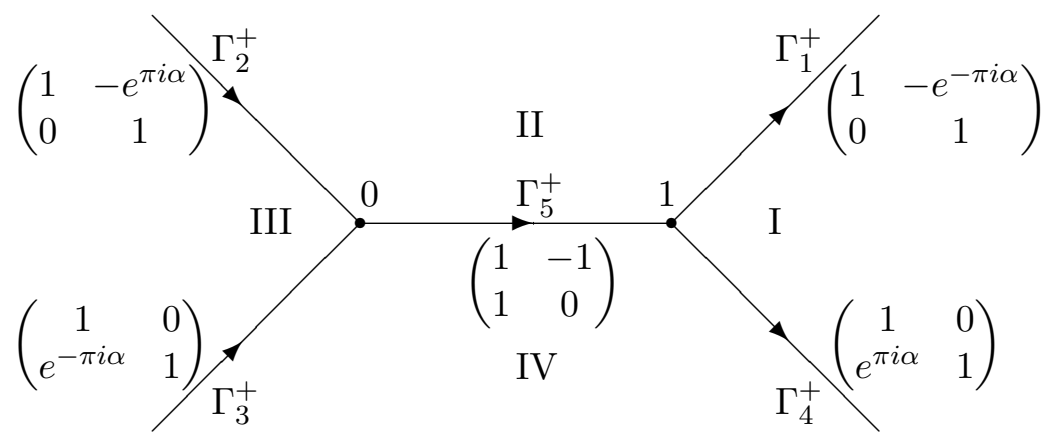

Figure 5. The jump contour $\Gamma^{+}$and the jump matrices for $\Psi^{+}$.

\section{Model RH problem for $\tau>0$}

The model RH problem relevant for the case $\tau>0$ is a special case of the one found in [5] (up to a simple transformation), and differs from the one for $\tau<0$, although there are similarities. For instance, the following $\mathrm{RH}$ problem, is also connected to the Painlevé V equation, and an explicit solution can be constructed for even $\alpha$ (but not for odd $\alpha$, which was possible when $\tau<0$ ). In the RH problem below, the relevant values for the parameters are $\alpha>-1 / 2$ and $s \in(0,-i \infty)$.

\section{RH problem for $\Psi^{+}$}

(a) $\Psi^{+}: \mathbb{C} \backslash \Gamma^{+} \rightarrow \mathbb{C}^{2 \times 2}$ is analytic, with $\Gamma^{+}=\Gamma_{1}^{+} \cup \Gamma_{2}^{+} \cup \Gamma_{3}^{+} \cup \Gamma_{4}^{+} \cup[0,1]$, and

$$
\Gamma_{1}^{+}=1+e^{\frac{\pi i}{4}} \mathbb{R}^{+}, \quad \Gamma_{2}^{+}=e^{\frac{3 \pi i}{4}} \mathbb{R}^{+}, \quad \Gamma_{3}^{+}=e^{-\frac{3 \pi i}{4}} \mathbb{R}^{+}, \quad \Gamma_{4}^{+}=1+e^{-\frac{\pi i}{4}} \mathbb{R}^{+},
$$

oriented as in Fig. 5.

(b) $\Psi^{+}$has continuous boundary values $\Psi_{ \pm}^{+}$on $\Gamma^{+} \backslash\{0,1\}$. We have the jump relations

$$
\begin{array}{ll}
\Psi_{+}^{+}(z)=\Psi_{-}^{+}(z)\left(\begin{array}{cc}
1 & -e^{-\pi i \alpha} \\
0 & 1
\end{array}\right) & \text { for } z \in \Gamma_{1}^{+}, \\
\Psi_{+}^{+}(z)=\Psi_{-}^{+}(z)\left(\begin{array}{cc}
1 & -e^{\pi i \alpha} \\
0 & 1
\end{array}\right) & \text { for } z \in \Gamma_{2}^{+}, \\
\Psi_{+}^{+}(z)=\Psi_{-}^{+}(z)\left(\begin{array}{cc}
1 & 0 \\
e^{-\pi i \alpha} & 1
\end{array}\right) & \text { for } z \in \Gamma_{3}^{+}, \\
\Psi_{+}^{+}(z)=\Psi_{-}^{+}(z)\left(\begin{array}{cc}
1 & 0 \\
e^{\pi i \alpha} & 1
\end{array}\right) & \text { for } z \in \Gamma_{4}^{+}, \\
\Psi_{+}^{+}(z)=\Psi_{-}^{+}(z)\left(\begin{array}{cc}
1 & -1 \\
1 & 0
\end{array}\right) & \text { for } z \in(0,1) .
\end{array}
$$

(c) There exist $q, r, p$ (depending on $s, \alpha$ but not on $z$ ) such that $\Psi^{+}(z)$ has the following limiting behavior as $z \rightarrow \infty$ :

$$
\Psi^{+}(z)=\left(I+\frac{1}{z}\left(\begin{array}{cc}
q & r \\
p & -q
\end{array}\right)+\mathcal{O}\left(z^{-2}\right)\right) \exp \left(-\frac{s}{2} z \sigma_{3}\right) .
$$

(d) If $\alpha \notin \mathbb{N}$ then the functions

$$
G(z):=\Psi^{+}(z)\left(\begin{array}{cc}
1 & g \\
0 & 1
\end{array}\right) z^{-\frac{\alpha}{2} \sigma_{3}}
$$




$$
H(z):=\Psi^{+}(z)\left(\begin{array}{cc}
1 & e^{-\pi i \alpha} g \\
0 & 1
\end{array}\right)(z-1)^{-\frac{\alpha}{2} \sigma_{3}}
$$

are bounded for $z$ near 0 and 1 respectively when $z$ is in region IV, with $g=\frac{e^{\pi i \alpha}-1}{2 i \sin (\pi \alpha)}$. We let $z^{\frac{\alpha}{2}},(z-1)^{\frac{\alpha}{2}}>0$ on the + side of $(1, \infty)$. If $\alpha \in \mathbb{N}$ then the functions

$$
\begin{aligned}
& G(z):=\Psi^{+}(z)\left(\begin{array}{cc}
1 & \frac{1-e^{\pi i \alpha}}{2 \pi i} \log z \\
0 & 1
\end{array}\right) z^{-\frac{\alpha}{2} \sigma_{3}} \\
& H(z):=\Psi^{+}(z)\left(\begin{array}{cc}
1 & \frac{e^{-\pi i \alpha}-1}{2 \pi i} \log (z-1) \\
0 & 1
\end{array}\right)(z-1)^{-\frac{\alpha}{2} \sigma_{3}}
\end{aligned}
$$

are bounded for $z$ near 0 and 1 respectively when $z$ is in region IV. We let $\log z, \log (z-1)$ $>0$ on the + side of $(1, \infty)$.

The above RH problem is equivalent to the one from [5, Section 3]. Indeed, if we define

$$
\Psi_{\mathrm{CK}}(\zeta ; s)=e^{\frac{s}{4} \sigma_{3}} \Psi^{+}\left(\frac{i}{2}(\zeta-i) ; s\right),
$$

then $\Psi_{\mathrm{CK}}$ solves the RH problem in [5, Section 3] in the case $\beta_{1}=\beta_{2}=0, \alpha_{1}=\alpha_{2}=\alpha / 2$.

In [5], it was proved that the RH problem for $\Psi_{\mathrm{CK}}$ is solvable if $\alpha>-1 / 2$ for every $s \in$ $(0,-i \infty)$, and it follows that the same is true for the $\mathrm{RH}$ problem for $\Psi^{+}$.

For $\tau>0$ and $u \in \mathbb{R}$, we define

$$
\left(\begin{array}{l}
\Phi_{1}(u ; \tau) \\
\Phi_{2}(u ; \tau)
\end{array}\right)=\Psi^{+}\left(z=\frac{2 u}{|s|}+\frac{1}{2} ; s\right) \Delta\left(\frac{2 u}{|s|}+\frac{1}{2}\right), \quad s=-i \sqrt{\tau},
$$

where

$$
\Delta(z)= \begin{cases}\left(\begin{array}{l}
e^{-\frac{\pi i \alpha}{2}} \\
-e^{\frac{\pi i \alpha}{2}}
\end{array}\right) & \text { for } \operatorname{Re} z>1, \\
\left(\begin{array}{c}
0 \\
-1
\end{array}\right) & \text { for } 0<\operatorname{Re} z<1, \\
\left(\begin{array}{c}
e^{\frac{\pi i \alpha}{2}} \\
-e^{-\frac{\pi i \alpha}{2}}
\end{array}\right) & \text { for } \operatorname{Re} z<0,\end{cases}
$$

and where $\Psi^{+}(z ; s)$ has to be understood as $\Psi_{+}^{+}(z ; s)$ for $z \in(0,1)$. Then, $\Phi_{1}(u ; \tau), \Phi_{2}(u ; \tau)$ are

smooth on the real line, except at the singularities $\pm \frac{\sqrt{\tau}}{4}$. As $u \rightarrow \pm \infty, \Phi_{1}$ and $\Phi_{2}$ have the asymptotic behavior

$$
\left(\begin{array}{l}
\Phi_{1}(u ; \tau) \\
\Phi_{2}(u ; \tau)
\end{array}\right)=\left(I+\mathcal{O}\left(u^{-1}\right)\right) \exp \left((i u+i \sqrt{\tau} / 4) \sigma_{3}\right) \Delta\left(\frac{2 u}{\sqrt{\tau}}+\frac{1}{2}\right)
$$

similar to $(2.4)$.

\subsection{Lax pair and Painlevé V}

The results about the Lax pair and the relation with the Painlevé V equation hold for $\Psi^{+}$as well as for $\Psi^{-}$. Indeed, $\Psi=\Psi^{+}$satisfies the Lax pair (2.5)-(2.6), where $A$ and $B$ can be parameterized as in (2.7)-(2.11). The functions $v, u$ defined in (2.12), (2.13) in terms of $q, r, p$, solve equations (2.14), (2.15), and $\sigma$ defined as in (2.18) solves the $\sigma$-form (1.10) of Painlevé V. It has to be noted that the functions $q(s), r(s), p(s), v(s), u(s), \sigma(s)$ defined here for $\tau>0$ are in general different from their counterparts in Section 2 for $\tau<0$. 


\subsection{Recursive construction for $\alpha \in 2 \mathbb{N}$}

Similarly to the case $\tau<0$, we define

$$
X_{\alpha}(z ; s)= \begin{cases}\Psi_{\alpha}^{+}(z ; s) & \text { for } z \in \mathrm{I}, \mathrm{III}, \\
\Psi_{\alpha}^{+}(z ; s)\left(\begin{array}{ll}
1 & 1 \\
0 & 1
\end{array}\right) & \text { for } z \in \mathrm{II}, \\
\Psi_{\alpha}^{+}(z ; s)\left(\begin{array}{ll}
1 & 0 \\
1 & 1
\end{array}\right) & \text { for } z \in \mathrm{IV},\end{cases}
$$

with regions I, II, III, IV as in Fig. 5. Then, it is easily verified that, for even $\alpha, X_{\alpha}$ satisfies the RH problem for $X_{\alpha}$ stated in Section 2.2. It is important to note that this is not the case for $\alpha$ odd. It follows that the formulas for $X_{2}$ and $q_{2}$ given in (2.35) and (2.36) still hold for $\tau>0$. By (2.37), we obtain the formula (1.14) for $\sigma_{2}^{+}$.

\subsection{The kernel $\mathbb{K}_{\alpha}$ as $\tau \rightarrow+\infty$}

An asymptotic analysis as $s=-i \sqrt{\tau} \rightarrow-i \infty$ of the $\mathrm{RH}$ problem for $\Psi_{\mathrm{CK}}$, equivalent to the $\mathrm{RH}$ problem for $\Psi^{+}$, has been performed in [5, Section 5]. The following result can be extracted from that analysis:

$$
\Psi_{+}^{+}(x ; s)\left(\begin{array}{ll}
1 & 1 \\
0 & 1
\end{array}\right) e^{\frac{s x}{2} \sigma_{3}}=I+\mathcal{O}\left(\frac{1}{s(|x|+1)}\right) \quad \text { for } x \in(0,1)
$$

as $s \rightarrow-i \infty$. Substituting (3.10) and (3.6) in (1.27), we obtain that the Painlevé kernel $\mathbb{K}_{\alpha}^{\mathrm{PV}}$ converges to the sine kernel, proving (1.28) whenever $|u-v|$ remains bounded in the limit where $\tau \rightarrow+\infty:$

$$
\mathbb{K}_{\alpha}^{\mathrm{PV}}(u, v ; \tau)=\mathbb{K}^{\sin }(u, v)+\mathcal{O}\left(\tau^{-1 / 2}\right) .
$$

\subsection{The kernel $\mathbb{K}_{\alpha}^{\mathrm{PV}}$ as $\tau \rightarrow 0$}

Recall that the parameter $s$ in the model problem for $\Psi^{+}$is negative imaginary. Define

$$
\begin{aligned}
& \Psi^{(2)}(z ; s)=e^{\frac{s}{4} \sigma_{3}} \Psi^{+}\left(\frac{2 z}{|s|}+\frac{1}{2} ; s\right) \chi(z), \\
& \chi(z)= \begin{cases}I, & \text { for }-\frac{|s|}{4}<\operatorname{Re} z<\frac{|s|}{4}, \\
e^{\frac{\pi i \alpha}{2}} \sigma_{3}, & \text { for } \operatorname{Re} z<-\frac{|s|}{4}, \\
e^{\frac{-\pi i \alpha}{2}} \sigma_{3}, & \text { for } \operatorname{Re} z>\frac{|s|}{4} .\end{cases}
\end{aligned}
$$

Then $\Psi^{(2)}$ satisfies the following $\mathrm{RH}$ problem:

\section{RH problem for $\Psi^{(2)}$}

(a) $\Psi^{(2)}: \mathbb{C} \backslash \widetilde{\Gamma}^{+} \rightarrow \mathbb{C}$ is analytic, where $\widetilde{\Gamma}^{+}$is as in Fig. 6,

$$
\begin{array}{ll}
\widetilde{\Gamma}^{+}=\cup_{i=1}^{8} \widetilde{\Gamma}_{i}^{+}, & \widetilde{\Gamma}_{1}^{+}=\{z: \arg (z-|s| / 4)=\pi / 4\}, \\
\widetilde{\Gamma}_{2}^{+}=\{z: \arg (z+|s| / 4)=3 \pi / 4\}, & \widetilde{\Gamma}_{3}^{+}=\{z: \arg (z+|s| / 4)=5 \pi / 4\}, \\
\widetilde{\Gamma}_{4}^{+}=\{z: \arg (z-|s| / 4)=7 \pi / 4\}, & \widetilde{\Gamma}_{5}^{+}=[-|s| / 4,|s| / 4], \\
\widetilde{\Gamma}_{6}^{+}=\{z: \operatorname{Re} z=|s| / 4\}, & \widetilde{\Gamma}_{7}^{+}=\{z: \operatorname{Re} z=-|s| / 4\} .
\end{array}
$$




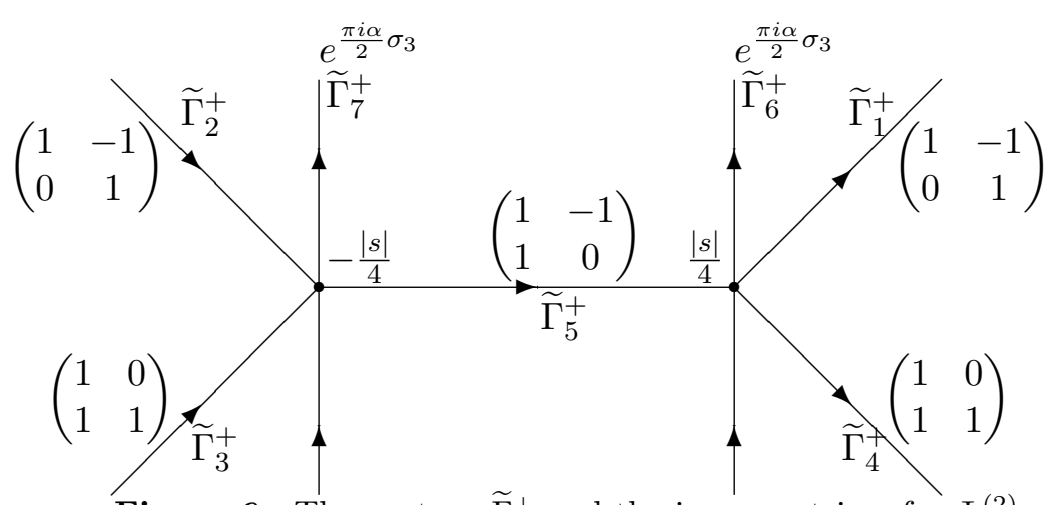

Figure 6. The contour $\widetilde{\Gamma}^{+}$and the jump matrices for $\Psi^{(2)}$.

(b) $\Psi^{(2)}$ has continuous boundary values on $\widetilde{\Gamma}^{+} \backslash\left\{-\frac{|s|}{4}, \frac{|s|}{4}\right\}$ given by

$$
\begin{array}{ll}
\Psi_{+}^{(2)}(z)=\Psi_{-}^{(2)}(z)\left(\begin{array}{cc}
1 & -1 \\
0 & 1
\end{array}\right) & \text { for } z \in \widetilde{\Gamma}_{1}^{+} \cup \widetilde{\Gamma}_{2}^{+}, \\
\Psi_{+}^{(2)}(z)=\Psi_{-}^{(2)}(z)\left(\begin{array}{cc}
1 & 0 \\
1 & 1
\end{array}\right) & \text { for } z \in \widetilde{\Gamma}_{3}^{+} \cup \widetilde{\Gamma}_{4}^{+}, \\
\Psi_{+}^{(2)}(z)=\Psi_{-}^{(2)}(z)\left(\begin{array}{cc}
1 & -1 \\
1 & 0
\end{array}\right) & \text { for } z \in \widetilde{\Gamma}_{5}^{+}, \\
\Psi_{+}^{(2)}(z)=\Psi_{-}^{(2)}(z) e^{\frac{\pi i \alpha}{2} \sigma_{3}} & \text { for } z \in \widetilde{\Gamma}_{6}^{+} \cup \widetilde{\Gamma}_{7}^{+} .
\end{array}
$$

(c) $\Psi^{(2)}(z)$ has the following asymptotic behavior as $z \rightarrow \infty$ :

$$
\Psi^{(2)}(z)=\left(I+\mathcal{O}\left(z^{-1}\right)\right) e^{i z \sigma_{3}} \chi(z) .
$$

(d) $\Psi^{(2)}(z)$ inherits its local behavior at $-\frac{|s|}{4}$ and $\frac{|s|}{4}$ from $\Psi^{+}(z)$ through (3.1)-(3.4) and (3.11).

When $s \rightarrow-i 0$ it was shown in [5, Section 6.2] that, for $z$ bounded away from 0 ,

$$
\Psi^{(2)}(z ; s) \chi^{-1}(z) M^{-1}(z)=I+\epsilon(z, s)
$$

where $M$ is as in $(2.47), \epsilon(z, s)=\mathcal{O}\left(|s|^{2}\right)+\mathcal{O}\left(|s|^{2+4 \alpha}\right)$ for $2 \alpha \notin \mathbb{Z}$, and $\epsilon(z, s)=\mathcal{O}(|s| \log |s|)$ for $2 \alpha \in \mathbb{Z}$.

We substitute (3.14) and (3.11) into (3.6) to find that as $\tau \rightarrow 0, \pm x>0$,

$$
\left(\begin{array}{l}
\Phi_{1}\left(x ;-s^{2}\right) \\
\Phi_{2}\left(x ;-s^{2}\right)
\end{array}\right)=M(x) e^{\mp \frac{\pi i \alpha}{2}}\left(\begin{array}{c}
1 \\
-1
\end{array}\right)+\epsilon(x, s) .
$$

By (1.27), we obtain the Bessel kernel after similar calculations as for the case $\tau<0$, proving (1.29).

\section{RH problem for orthogonal polynomials}

\subsection{RH problem for orthogonal polynomials and differential identity}

The correlation kernel (1.20) for the eigenvalues in the random matrix ensemble (1.1) can be expressed in terms of the solution of a $\mathrm{RH}$ problem which characterizes the orthogonal polynomials $p_{j}^{(n)}$ with respect to the weight $w_{n}$ defined in (1.3). 
Define $Y$ by

$$
Y(z ; n)=\left(\begin{array}{cc}
\frac{1}{\kappa_{n}^{(n)}} p_{n}^{(n)}(z) & \frac{1}{2 \pi i \kappa_{n}^{(n)}} \int_{\mathbb{R}} \frac{p_{n}^{(n)}(x)}{x-z} w_{n}(x) d x \\
-2 \pi i \kappa_{n-1}^{(n)} p_{n-1}^{(n)}(z) & -\kappa_{n-1}^{(n)} \int_{\mathbb{R}} \frac{p_{n-1}^{(n)}(x)}{x-z} w_{n}(x) d x
\end{array}\right),
$$

where $\kappa_{j}^{(n)}>0$ is the leading coefficient of the normalized orthogonal polynomial $p_{j}^{(n)}$. It is well-known [15] that $Y$ can be characterized as the unique solution of the following RH problem.

\section{RH problem for $Y$}

(a) $Y$ is analytic in $\mathbb{C} \backslash \mathbb{R}$.

(b) $Y$ has the following jump relations on $\mathbb{R}$, except at the singularities $\pm \sqrt{t}$ in the case where $t>0$ :

$$
Y_{+}(x)=Y_{-}(x)\left(\begin{array}{cc}
1 & w_{n}(x) \\
0 & 1
\end{array}\right)
$$

(c) As $z \rightarrow \infty$,

$$
Y(z)=\left(I+\mathcal{O}\left(z^{-1}\right)\right)\left(\begin{array}{cc}
z^{n} & 0 \\
0 & z^{-n}
\end{array}\right)
$$

(d) If $t>0$ and $\alpha<0, Y(z)$ has the behavior

$$
Y(z)=\left(\begin{array}{ll}
\mathcal{O}(1) & \mathcal{O}\left(|z \mp \sqrt{t}|^{\alpha}\right) \\
\mathcal{O}(1) & \mathcal{O}\left(|z \mp \sqrt{t}|^{\alpha}\right)
\end{array}\right)
$$

as $z \rightarrow \pm \sqrt{t}$. If $t>0$ and $\alpha \geq 0, Y$ is bounded near $\pm \sqrt{t}$.

We can express the eigenvalue correlation kernel $K_{n}$ and the logarithmic $t$-derivative of the partition function $\widehat{Z}_{n}$ exactly in terms of $Y$.

\section{Proposition 4.1.}

(1) Let $K_{n}$ be the correlation kernel defined in (1.20). For $x, y \in \mathbb{R}$, we have

$$
K_{n}(x, y)=\frac{\sqrt{w_{n}(x) w_{n}(y)}}{2 \pi i} \frac{\left(\begin{array}{ll}
0 & 1
\end{array}\right) Y_{+}^{-1}(y) Y_{+}(x)\left(\begin{array}{l}
1 \\
0
\end{array}\right)}{x-y} .
$$

(2) Let $\widehat{Z}_{n}(t)=\widehat{Z}_{n}(t, \alpha, V)$ be the partition function defined in (1.4). Write $z_{0}=\sqrt{t}$ when $t>0$ and $z_{0}=i \sqrt{-t}$ when $t<0$. For $t<0, \alpha>-\frac{1}{2}$ and for $t>0, \alpha \geq 0$, we have the differential identity

$$
\frac{d}{d t} \log \widehat{Z}_{n}(t)=-\frac{\alpha}{2 z_{0}}\left(\left(Y^{-1} \frac{d Y}{d z}\right)_{22}\left(z_{0}\right)-\left(Y^{-1} \frac{d Y}{d z}\right)_{22}\left(-z_{0}\right)\right)
$$


Proof. The formula for the correlation kernel (4.2) follows after a straightforward calculation from (1.20) and (4.1).

To obtain (4.3), we follow ideas from [25] and [5]. In the remaining part of this proof, we suppress the dependence on $n$ in our notations and we write $p_{j}, \kappa_{j}$, and $w$ instead of $p_{j}^{(n)}, \kappa_{j}^{(n)}$, and $w_{n}$. We start with the well known formula

$$
\widehat{Z}_{n}(t)=\prod_{j=0}^{n-1} \kappa_{j}^{-2} .
$$

Using the orthogonality of the polynomials and the Christoffel-Darboux formula, it follows that

$$
\begin{aligned}
\frac{d}{d t} \log \widehat{Z}_{n}(t) & =-2 \sum_{j=0}^{n-1} \frac{\partial_{t} \kappa_{j}}{\kappa_{j}}=-2 \sum_{j=0}^{n-1} \int_{-\infty}^{\infty} p_{j}(x) \partial_{t}\left(p_{j}(x)\right) w(x) d x \\
& =-\int_{-\infty}^{\infty} \partial_{t}\left(\sum_{j=0}^{n-1} p_{j}^{2}(x)\right) w(x) d x \\
& =-\int_{-\infty}^{\infty} \partial_{t}\left(\frac{\kappa_{n-1}}{\kappa_{n}}\left(\partial_{x}\left(p_{n}(x)\right) p_{n-1}(x)-p_{n}(x) \partial_{x}\left(p_{n-1}(x)\right)\right)\right) w(x) d x
\end{aligned}
$$

By orthogonality, it is straightforward to check that

$$
\begin{aligned}
& \frac{d}{d t} \log \widehat{Z}_{n}(t)=-n \frac{\partial_{t} \kappa_{n-1}}{\kappa_{n-1}}+\frac{\kappa_{n-1}}{\kappa_{n}}\left(J_{1}-J_{2}\right), \\
& J_{1}=\int_{-\infty}^{\infty} \partial_{t} p_{n}(x) \partial_{x} p_{n-1}(x) w(x) d x, \quad J_{2}=\int_{-\infty}^{\infty} \partial_{x} p_{n}(x) \partial_{t} p_{n-1}(x) w(x) d x .
\end{aligned}
$$

We start by evaluating $J_{1}$ :

$$
J_{1}=-\int_{-\infty}^{\infty} p_{n}(x) \partial_{t}\left(\partial_{x} p_{n-1}(x) w(x)\right) d x+\int_{-\infty}^{\infty} \partial_{t}\left(p_{n}(x) \partial_{x} p_{n-1}(x) w(x)\right) d x .
$$

The rightmost term in this expression vanishes, as one can see by taking the derivative outside the integral and using orthogonality. By the form of the weight function $w$ given in (1.3), we obtain

$$
\begin{aligned}
& J_{1}=\frac{\alpha}{2 z_{0}}\left(I_{+}^{(1)}-I_{-}^{(1)}\right), \\
& I_{ \pm}^{(1)}=\int_{-\infty}^{\infty} \frac{p_{n}(x) \partial_{x} p_{n-1}(x) w(x)}{x \mp z_{0}} d x .
\end{aligned}
$$

Writing $\left.\partial_{x}\left(p_{m}(x)\right)\right|_{x=y}=p_{m, x}(y)$, we find that

$$
\begin{aligned}
I_{ \pm}^{(1)} & =\int_{-\infty}^{\infty} \frac{p_{n}(x)\left(p_{n-1, x}(x)-p_{n-1, x}\left( \pm z_{0}\right)\right) w(x)}{x \mp z_{0}} d x+p_{n-1, x}\left( \pm z_{0}\right) \int_{-\infty}^{\infty} \frac{p_{n}(x) w(x)}{x \mp z_{0}} d x \\
& =p_{n-1, x}\left( \pm z_{0}\right) \int_{-\infty}^{\infty} \frac{p_{n}(x) w(x)}{x \mp z_{0}} d x .
\end{aligned}
$$

We proceed in a similar fashion for $J_{2}$ and find that

$$
\begin{aligned}
J_{2}= & -n \frac{\kappa_{n} \partial_{t} \kappa_{n-1}}{\kappa_{n-1}^{2}}+\frac{\alpha}{2 z_{0}} p_{n, x}\left(z_{0}\right) \int_{-\infty}^{\infty} \frac{p_{n-1}(x) w(x)}{x-z_{0}} d x \\
& -\frac{\alpha}{2 z_{0}} p_{n, x}\left(-z_{0}\right) \int_{-\infty}^{\infty} \frac{p_{n-1}(x) w(x)}{x+z_{0}} d x .
\end{aligned}
$$

Substituting (4.7)-(4.9) into (4.4), and using (4.1), it is straightforward to obtain (4.3). 
Remark 4.2. When $t>0$ and $\alpha<0$, the integrals in (4.8) are not defined. Using techniques similar to those in [25], one can regularize those integrals and obtain a differential identity similar to (4.3). We refer the reader to $[5,25]$ for more details.

\subsection{The $g$-function and normalization of the $\mathrm{RH}$ problem}

We proceed with a transformation of the $\mathrm{RH}$ problem for $Y$ to one which is normalized to the identity at infinity, and which has suitable jump conditions. This is a standard part of the Deift/Zhou steepest descent method [13] which we apply in order to get asymptotics for $Y$ as $n \rightarrow \infty$ with $t$ small. Define

$$
T(z)=e^{\frac{n \ell}{2} \sigma_{3}} Y(z) e^{-n g(z) \sigma_{3}} e^{\frac{-n \ell}{2} \sigma_{3}},
$$

where $g$ is defined by

$$
g(z)=\int \log (z-x) d \mu_{V}(x)
$$

with $\mu_{V}$ the equilibrium measure given by (1.9), minimizing (1.6) and satisfying the conditions (1.7), (1.8). Clearly $g(z)=\log z+\mathcal{O}\left(z^{-1}\right)$ as $z \rightarrow \infty$, and it follows that $T$ satisfies the following $\mathrm{RH}$ problem.

\section{RH problem for $T$}

(a) $T$ is analytic in $\mathbb{C} \backslash \mathbb{R}$.

(b) $T$ has the following jump relation for $x \in \mathbb{R}$ (except for $x= \pm \sqrt{t}$ if $t>0$ ),

$$
T_{+}(x)=T_{-}(x)\left(\begin{array}{cc}
e^{-n\left(g_{+}(x)-g_{-}(x)\right)} & \left|x^{2}-t\right|^{\alpha} e^{n\left(g_{+}(x)+g_{-}(x)-V(x)+\ell\right)} \\
0 & e^{n\left(g_{+}(x)-g_{-}(x)\right)}
\end{array}\right) .
$$

(c) $T(z)=I+\mathcal{O}\left(z^{-1}\right)$ as $z \rightarrow \infty$.

(d) If $t>0, \alpha<0$, as $z \rightarrow \pm \sqrt{t}$, we have

$$
T(z)=\left(\begin{array}{ll}
\mathcal{O}(1) & \mathcal{O}\left(|z \mp \sqrt{t}|^{\alpha}\right) \\
\mathcal{O}(1) & \mathcal{O}\left(|z \mp \sqrt{t}|^{\alpha}\right)
\end{array}\right)
$$

If $t>0$ and $\alpha \geq 0, T$ is bounded near $\pm \sqrt{t}$.

By the fact that $V$ is one-cut regular, we have (1.9) with $h$ real analytic and positive on $[a, b]$. By (4.11) and the Euler-Lagrange conditions (1.7), (1.8), it can be shown that $g$ satisfies the following properties which we will need later on:

(a) $g_{+}(x)-g_{-}(x)= \begin{cases}0 & \text { for } x>b, \\ 2 \pi i & \text { for } x<a, \\ 2 \pi i \int_{x}^{b} h(\xi)((\xi-a)(b-\xi))^{\frac{1}{2}} d \xi & \text { for } x \in[a, b],\end{cases}$

(b) $g_{+}(x)+g_{-}(x)-V(x)+\ell<0$ for $x>b$ and $x<a$,

(c) $g_{+}(x)+g_{-}(x)-V(x)+\ell= \begin{cases}2 \pi \int_{x}^{a} h(\xi)((\xi-a)(\xi-b))^{1 / 2} d \xi & \text { for } x<a, \\ 0 & \text { for } x \in[a, b], \\ -2 \pi \int_{b}^{x} h(\xi)((\xi-a)(\xi-b))^{1 / 2} d \xi & \text { for } x>b .\end{cases}$ 


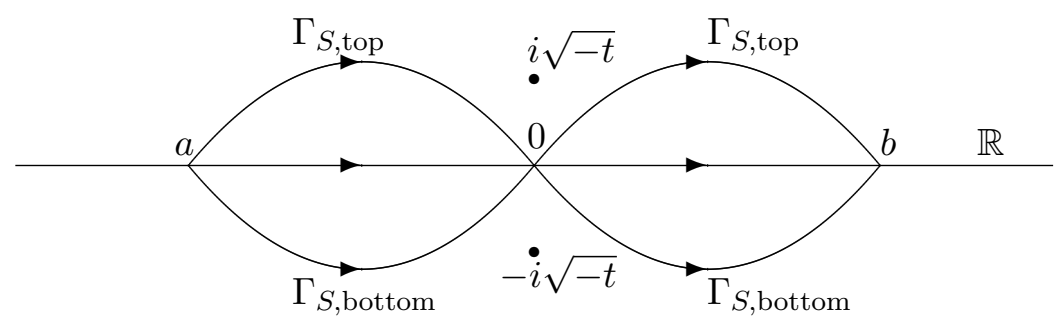

Figure 7. The contour $\Gamma_{S}$.

In part (a), the square root is positive for $s \in(a, b)$, and in part (c), the square roots are positive for $s>b$ and negative for $s<a$, with branch cut on $[a, b]$. The function $h$ is analytic on a neighborhood $\mathcal{U}$ of $\mathbb{R}$, and we define the function $\phi: \mathcal{U} \backslash(-\infty, b] \rightarrow \mathbb{C}$ as

$$
\phi(z)=\pi \int_{b}^{z} h(\xi)((\xi-a)(\xi-b))^{1 / 2} d \xi
$$

with the square root analytic on $\mathbb{C} \backslash[a, b]$ and positive for $\xi>b$, and with the path of integration not crossing $(-\infty, b]$. By property (a) of the function $g$, it follows that

$$
\mp\left(g_{+}(x)-g_{-}(x)\right)=2 \phi_{ \pm}(x) \quad \text { for } x \in(a, b) \text {. }
$$

Combining this with property (c) for the function $g$ we obtain

$$
T_{+}(x)=T_{-}(x)\left(\begin{array}{cc}
e^{2 n \phi_{+}(x)} & \left|x^{2}-t\right|^{\alpha} \\
0 & e^{2 n \phi_{-}(x)}
\end{array}\right) \quad \text { for } x \in[a, b] .
$$

By property (c) of the function $g$ it follows that

$$
g_{+}(x)+g_{-}(x)-V(x)+\ell= \begin{cases}-2 \phi(x) & \text { for } x>b, \\ -2 \phi_{+}(x)-2 \pi i & \text { for } x<a .\end{cases}
$$

Combined with property (a) of $g$ it follows that

$$
T_{+}(x)=T_{-}(x)\left(\begin{array}{cc}
1 & \left|x^{2}-t\right|^{\alpha} e^{-2 n \phi(x)} \\
0 & 1
\end{array}\right) \quad \text { for } x<a \text { and for } b<x \text {, }
$$

where we note that $e^{-2 n \phi_{+}(x)}=e^{-2 n \phi_{-}(x)}$ by (4.14).

\section{Asymptotic analysis for $T$ as $n \rightarrow \infty$ for $t<0$}

In what follows, we will perform further transformations on $T$ in order to obtain a $\mathrm{RH}$ problem for which we can compute large $n$ asymptotics. We first complete the analysis in the case $t<0$.

\subsection{Opening of the lens}

We deform the jump contour for $T$, which is the real line, to a lens-shaped contour around $[a, b]$ as in Fig. 7. It is important that the singularities $\pm i \sqrt{-t}$ lie in the region outside of the lens, and that the lenses lie within the region $\mathcal{U}$ of analyticity of $h$. Since we want to obtain asymptotics which are valid for $t$ arbitrary small, we choose the lenses to pass through 0 , and with shape independent of $t$ and $n$. 
Define

$$
S(z)= \begin{cases}T(z) & \text { for } z \text { outside the lens, } \\
T(z)\left(\begin{array}{cc}
1 & 0 \\
-\frac{e^{2 n \phi(z)}}{\left(z^{2}-t\right)^{\alpha}} & 1
\end{array}\right) & \text { for } z \text { in the upper parts of the lens } \\
T(z)\left(\begin{array}{cc}
1 & 0 \\
\frac{e^{2 n \phi(z)}}{\left(z^{2}-t\right)^{\alpha}} & 1
\end{array}\right) & \text { for } z \text { in the lower parts of the lens. }\end{cases}
$$

The function $\left(z^{2}-t\right)^{\alpha}$ is chosen with branch cuts on $(-i \infty,-i \sqrt{-t}] \cup[i \sqrt{-t},+i \infty)$, outside the lens-shaped region, and positive for real $z$. It is such that $\left(z^{2}-t\right)^{\alpha}=\left|z^{2}-t\right|^{\alpha}$ for $z \in(a, b)$.

It follows that $S$ solves the following $\mathrm{RH}$ problem.

\section{RH problem for $S$}

(a) $S$ is analytic for $z \in \mathbb{C} \backslash \Gamma_{S}$ where $\Gamma_{S}=\Gamma_{S \text {,top }} \cup \Gamma_{S \text {,bottom }} \cup \mathbb{R}$ is the contour shown in Fig. 7 .

(b) $S$ has the following jump relations on $\Gamma_{S}$ :

$$
\begin{array}{ll}
S_{+}(x)=S_{-}(x)\left(\begin{array}{cc}
1 & \left|x^{2}-t\right|^{\alpha} e^{-2 n \phi(x)} \\
0 & 1
\end{array}\right), & \text { for } x \in \mathbb{R} \backslash[a, b], \\
S_{+}(x)=S_{-}(x)\left(\begin{array}{cc}
0 & \left|x^{2}-t\right|^{\alpha} \\
-\frac{1}{\left|x^{2}-t\right|^{\alpha}} & 0
\end{array}\right), & \text { for } x \in(a, b), \\
S_{+}(z)=S_{-}(z)\left(\begin{array}{cc}
1 & 0 \\
\frac{e^{2 n \phi(z)}}{\left(z^{2}-t\right)^{\alpha}} & 1
\end{array}\right), & \text { for } z \in \Gamma_{S, \text { top }} \cup \Gamma_{S, \text { bottom }} .
\end{array}
$$

(c) $S(z)=I+\mathcal{O}\left(z^{-1}\right)$ as $z \rightarrow \infty$.

(d) $S(z)$ is bounded as $z \rightarrow 0$, as $z \rightarrow a$, and as $z \rightarrow b$.

\subsection{Global parametrix for $t<0$}

The jump matrices for $S$ tend to the identity matrix exponentially fast everywhere on $\Gamma_{S}$, with the exception of the interval $(a, b)$ and small neighborhoods of $a, b$, and 0 . We construct a global parametrix $N$ which solves the RH problem for $S$, ignoring exponentially small jumps and small neighborhoods of $a, b$, and 0 . We will show later on that the global parametrix is a good approximation of $S$ away from $a, b, 0$.

\section{RH problem for $N$}

(a) $N: \mathbb{C} \backslash[a, b] \rightarrow \mathbb{C}^{2 \times 2}$ is analytic.

(b) $N$ has the following jump relation on $(a, b)$, where the orientation is taken from left to right:

$$
N_{+}(x)=N_{-}(x)\left(\begin{array}{cc}
0 & \left|x^{2}-t\right|^{\alpha} \\
-\frac{1}{\left|x^{2}-t\right|^{\alpha}} & 0
\end{array}\right) \quad \text { for } x \in(a, b) .
$$

(c) $N(z)=I+\mathcal{O}\left(\frac{1}{z}\right)$ as $z \rightarrow \infty$. 
Define

$$
N(z)=D(\infty)^{-1} N_{0}(z) D(z),
$$

where

$$
N_{0}(z)=\left(\begin{array}{cc}
1 & i \\
i & 1
\end{array}\right)\left(\begin{array}{cc}
\gamma(z) & 0 \\
0 & \gamma(z)^{-1}
\end{array}\right)\left(\begin{array}{cc}
1 & i \\
i & 1
\end{array}\right)^{-1}, \quad \gamma(z)=\left(\frac{z-b}{z-a}\right)^{1 / 4}
$$

with $\left(\frac{z-b}{z-a}\right)^{1 / 4}$ analytic on $\mathbb{C} \backslash[a, b]$ and positive for $z>b . D(z)$ depends on $\alpha$ and $t$ and is given by

$$
\begin{aligned}
& D(z)=d(z)^{-\sigma_{3}}, \\
& d(z)=\exp \left(-\alpha \frac{((z-a)(z-b))^{1 / 2}}{2 \pi}\left(\int_{a}^{b} \frac{\log \left|\xi^{2}-t\right| d \xi}{(\xi-z) \sqrt{(\xi-a)(b-\xi)}}\right)\right),
\end{aligned}
$$

with $\sqrt{(z-a)(z-b)}$ analytic in $\mathbb{C} \backslash[a, b]$ and positive for $z>b$, and with $\sqrt{(\xi-a)(b-\xi)}$ positive for $\xi \in(a, b)$. Then, it is straightforward to verify that $N$ solves the RH problem for $N$. Moreover, as $z \rightarrow a$ and $z \rightarrow b, N(z)$ has the following behavior:

$$
\begin{array}{ll}
N(z)=\mathcal{O}\left(|z-a|^{-1 / 4}\right) & \text { as } z \rightarrow a, \\
N(z)=\mathcal{O}\left(|z-b|^{-1 / 4}\right) & \text { as } z \rightarrow b .
\end{array}
$$

To verify this, one first shows that $N_{0}(z)$ satisfies the conditions (5.6), (5.7). It then remains to show that $d$ is bounded near $a$ and $b$. This can be seen by deforming the integral between $a$ and $b$ to a contour surrounding the interval $[a, b]$ and applying a residue argument.

\subsection{Local parametrix at $a$ and $b$}

We need to construct local parametrices near the endpoints $a$ and $b$. This construction, using the Airy function, is standard $[7,11,12]$. The explicit formula for the local parametrices near $a$ and $b$ is not relevant to later calculations, we only need that these local parametrices exist.

Let $U_{b}\left(U_{a}\right)$ be an open set which is independent of $n$ and which contains $b(a)$. The following $\mathrm{RH}$ problems can be solved in terms of the Airy function. We do not give details about this construction here.

\section{RH problem for $\boldsymbol{P}^{(b)}$}

(a) $P^{(b)}: U_{b} \backslash \Gamma_{S} \rightarrow \mathbb{C}^{2 \times 2}$ is analytic on $U_{b} \backslash \Gamma_{S}$.

(b) $P^{(b)}$ has exactly the same jump relations as $S$ has for $z \in \Gamma_{S} \cap U_{b}$.

(c) $P^{(b)}(z)=\left(I+\mathcal{O}\left(n^{-1}\right)\right) N(z)$ as $n \rightarrow \infty$, uniformly for $z \in \partial U_{b}$ and $|t|$ sufficiently small.

\section{RH problem for $P^{(a)}$}

(a) $P^{(a)}: U_{a} \backslash \Gamma_{S} \rightarrow \mathbb{C}^{2 \times 2}$ is analytic on $U_{a} \backslash \Gamma_{S}$.

(b) $P^{(a)}$ has exactly the same jump relations as $S$ has for $z \in \Gamma_{S} \cap U_{a}$.

(c) $P^{(a)}(z)=\left(I+\mathcal{O}\left(n^{-1}\right)\right) N(z)$ as $n \rightarrow \infty$, uniformly for $z \in \partial U_{a}$ and $|t|$ sufficiently small. 


\subsection{Local parametrix at the origin}

Let $U_{0}$ be a sufficiently small disk around the origin, which is independent of $n$. We want to construct a local parametrix which has the same jumps as $S$ in the disk $U_{0}$ and which matches with the global parametrix up to order $1 / n$ at the boundary of $U_{0}$. This matching condition has to be valid in the double scaling limit where $n \rightarrow \infty$ and simultaneously $t \rightarrow 0$ in such a way that $n^{2} t$ tends to a non-zero constant, or equivalently such that $\tau_{n, t} \rightarrow \tau<0$, with $\tau_{n, t}$ defined in (1.23).

\section{RH problem for $P$}

(a) $P: U_{0} \backslash \Gamma_{S} \rightarrow \mathbb{C}^{2 \times 2}$ is analytic.

(b) $P$ has the same jump relations as $S$ :

$$
P_{-}^{-1}(z) P_{+}(z)=S_{-}^{-1}(z) S_{+}(z) \quad \text { for } z \in U_{0} \cap \Gamma_{S} .
$$

(c) $P(z)=\left(I+\mathcal{O}\left(n^{-1}\right)\right) N(z)$ uniformly for $z$ on the boundary $\partial U_{0}$, as $n \rightarrow \infty$ and simultaneously $t \rightarrow 0$ in such a way that $n^{2} t$ tends to a non-zero constant.

We will construct a solution to the $\mathrm{RH}$ problem for $P$ in terms of the model $\mathrm{RH}$ solution $\Psi^{-}$ studied in Section 2.

\subsubsection{Construction of the parametrix}

Define $\lambda: U_{0} \rightarrow \mathbb{C}$ by

$$
\lambda(z)=-i n \times \begin{cases}\phi(z)-\frac{\phi(i \sqrt{-t})-\phi(-i \sqrt{-t})}{2} & \text { for } \operatorname{Im} z>0, \\ -\phi(z)-\frac{\phi(i \sqrt{-t})-\phi(-i \sqrt{-t})}{2} & \text { for } \operatorname{Im} z<0 .\end{cases}
$$

By (4.13), $\lambda$ is a conformal map in $U_{0}$ (if this one is chosen sufficiently small, but containing $\pm i \sqrt{-t}$ ), and we have

$$
\lambda(i \sqrt{-t})=-\lambda(-i \sqrt{-t})=-\frac{i n}{2}(\phi(i \sqrt{-t})+\phi(-i \sqrt{-t})),
$$

and

$$
\lambda^{\prime}(0)=n \pi \psi_{V}(0) .
$$

We define $s_{n, t}$ such that $\lambda$ sends $i \sqrt{-t}$ to $i s_{n, t} / 4$ and $-i \sqrt{-t}$ to $-i s_{n, t} / 4$ :

$$
s_{n, t}=-4 i \lambda(i \sqrt{-t})=-2 n(\phi(i \sqrt{-t})+\phi(-i \sqrt{-t}))=-4 n \operatorname{Re} \phi(i \sqrt{-t})>0 .
$$

By (4.13), we have

$$
\begin{aligned}
s_{n, t} & =-4 \pi n \operatorname{Re}\left(\int_{0}^{i \sqrt{-t}} h(s)((s-a)(s-b))^{1 / 2} d s\right) \\
& =4 n \pi \sqrt{-t} \psi_{V}(0)+\mathcal{O}\left(n|t|^{3 / 2}\right)=\sqrt{-\tau_{n, t}}+\mathcal{O}\left(n|t|^{3 / 2}\right),
\end{aligned}
$$

as $t \rightarrow 0, n \rightarrow \infty$, with $\tau_{n, t}$ as in (1.23).

There remains some freedom to choose the contour $\Gamma_{S}$, which has to be of the shape shown in Fig. 7, but is otherwise arbitrary. We choose $\Gamma_{S}$ such that the upper and lower lenses are mapped to the straight lines $\widetilde{\Gamma}_{1}^{-}, \ldots, \widetilde{\Gamma}_{4}^{-}$(see Fig. 3) by $\lambda$. 
Recall the definition of $\Psi^{(1)}$ from (2.42). We take $P$ of the form

$$
P(z)=E(z) \Psi^{(1)}\left(\lambda(z) ; s_{n, t}\right) W(z) .
$$

Here, $E$ has to be analytic in $U_{0}$, and $W$ takes the form

$$
W(z)= \begin{cases}\left(\left(z^{2}-t\right)^{\alpha / 2} e^{-n \phi(z)}\right)^{\sigma_{3}} \sigma_{3} \sigma_{1} & \text { for } \operatorname{Im} z>0 \\ \left(\left(z^{2}-t\right)^{-\alpha / 2} e^{n \phi(z)}\right)^{\sigma_{3}} & \text { for } \operatorname{Im} z<0 .\end{cases}
$$

It is constructed in such a way that $P$ has the required jump conditions on $\Gamma_{S} \cap U_{0}$. The branch cuts of $\left(z^{2}-t\right)^{\alpha / 2}$ are taken on the lines $(-i \infty,-i \sqrt{-t})$ and $(i \sqrt{-t}, i \infty) . E$ is an analytic matrix-valued function on $U_{0}$ which is constructed such that $P(z)$ approximates $N(z)$ on the boundary $\partial U_{0}$ :

$$
E(z)=N(z) W^{-1}(z)\left(\frac{\lambda(z)-\frac{i s_{n, t}}{4}}{\lambda(z)+\frac{i s_{n, t}}{4}}\right)^{\frac{\alpha}{2} \sigma_{3}} e^{-i \lambda(z) \sigma_{3}} e^{\frac{\pi i \alpha}{2} \sigma_{3}},
$$

where we have chosen the branch cuts for $z$ in $(i \sqrt{-t}, i \infty)$ and $(-i \sqrt{-t},-i \infty)$ such that

$$
\left(\frac{\lambda(z)-\frac{i s_{n, t}}{4}}{\lambda(z)+\frac{i s_{n, t}}{4}}\right)^{\frac{\alpha}{2} \sigma_{3}}=1+\mathcal{O}\left(n^{-1}\right) \quad \text { for } \operatorname{Re}(z)>0 \text { and } z \in \partial U \text {, }
$$

as $n \rightarrow \infty$ and $s_{n, t}$ remains bounded. Note that $N(z) W^{-1}(z)$ is continuous across the real line and that $W^{-1}\left(\frac{\lambda(z)-\frac{i s_{n, t}}{4}}{\lambda(z)+\frac{i s_{n, t}}{4}}\right)^{\frac{\alpha}{2} \sigma_{3}}$ has no singularities at $\pm i \sqrt{-t}$. No other component of $E$ has discontinuities. It follows that $E$ is analytic on $U_{0}$.

Proposition 5.1. Let $P(z)$ be defined as in (5.11). Then, $P$ satisfies the $R H$ problem for $P$.

Proof. Conditions (a) and (b) are satisfied by construction. We verify (c). For $z \in \partial U_{0}$ we have

$$
P(z) N^{-1}(z)=E(z) \Psi^{(1)}\left(\lambda(z) ; s_{n, t}\right) W(z) N^{-1}(z) .
$$

It follows from (2.44) that

$$
\Psi^{(1)}\left(\lambda(z) ; s_{n, t}\right)\left(\frac{\lambda(z)-\frac{i s_{n, t}}{4}}{\lambda(z)+\frac{i s_{n, t}}{4}}\right)^{\frac{\alpha}{2} \sigma_{3}} e^{-i \lambda(z) \sigma_{3}} e^{\frac{\pi i \alpha}{2} \sigma_{3}}=I+\mathcal{O}\left(n^{-1}\right),
$$

as $n \rightarrow \infty$, where $\mathcal{O}\left(n^{-1}\right)$ is uniform for $s_{n, t}$ in compact subsets of $(0, \infty)$ and $z \in \partial U_{0}$ and branches are chosen as in (5.14). By (5.12), we find that

$$
P(z) N^{-1}(z)=E(z)\left(I+\mathcal{O}\left(n^{-1}\right)\right) E^{-1}(z) .
$$

The proposition follows if $E(z)$ is bounded uniformly on $\partial U_{0}$. By (5.13) it is clear that the only part of $E$ which could diverge is $W^{-1}(z) e^{-i \lambda(z) \sigma_{3}}$, which contains $e^{n \phi(z)-i \lambda(z)}$. To deal with this we note that

$$
e^{n \phi(z)-i \lambda(z)}=e^{-\frac{n}{2}(\phi(i \sqrt{-t})-\phi(-i \sqrt{-t}))},
$$

which has modulus 1 because of the symmetry $\overline{\phi(\bar{z})}=\phi(z)$. Substituting this into (5.13), one indeed finds that $E(z)=\mathcal{O}(1)$ uniformly on the boundary $\partial U_{0}$. 


\subsection{Small norm $\mathrm{RH}$ problem}

Define $R(z)$ by

$$
R(z)= \begin{cases}S(z) P(z)^{-1} & \text { for } z \in U_{0}, \\ S(z) P^{(a)}(z)^{-1} & \text { for } z \in U_{a} \\ S(z) P^{(b)}(z)^{-1} & \text { for } z \in U_{b} \\ S(z) N(z)^{-1} & \text { for } z \in \mathbb{C} \backslash\left(U_{0} \cup U_{a} \cup U_{b}\right) .\end{cases}
$$

It follows from the conditions of the RH problems for $P, P^{(a)}, P^{(b)}$ and $N$ that $R(z)$ satisfies

\section{RH problem for $R$}

(a) $R$ is analytic on $\mathbb{C} \backslash \Gamma_{R}$ where

$$
\left.\Gamma_{R}=\partial U_{a} \cup \partial U_{b} \cup \partial U_{0} \cup\left(\Gamma_{S} \backslash\left((a, b) \cup U_{0} \cup U_{a} \cup U_{b}\right)\right)\right) .
$$

(b) $R$ has the following jump relations on $\Gamma_{R}$ :

$$
R_{+}(z)=R_{-}(z)\left(I+\mathcal{O}\left(n^{-1}\right)\right)
$$

as $n \rightarrow \infty$, uniformly for $z \in \Gamma_{R}$, in the double scaling limit where $\tau_{n, t} \rightarrow \tau<0$.

(c) $R(z)$ has the following asymptotics as $z \rightarrow \infty$ :

$$
R(z)=I+\mathcal{O}\left(z^{-1}\right) .
$$

We have that $R$ solves a $\mathrm{RH}$ problem, normalized at infinity and with jump matrices that are uniformly close to the identity matrix. This type of $\mathrm{RH}$ problem is often called a small norm $\mathrm{RH}$ problem, and it implies that $R$ itself is uniformly close to $I$ : in the double scaling limit where $n \rightarrow \infty$ and $t \rightarrow 0$ in such a way that $\tau_{n, t} \rightarrow \tau<0$, we have

$$
R(z)=I+\mathcal{O}\left(n^{-1}\right)
$$

where the error term $\mathcal{O}\left(n^{-1}\right)$ is uniform for $z \in \mathbb{C} \backslash \Gamma_{R}$.

Remark 5.2. In fact, it can be shown that the estimate (5.20) holds not only if $n \rightarrow \infty, t \rightarrow 0$ in such a way that $\tau_{n, t} \rightarrow \tau<0$, but also if $\tau_{n, t} \rightarrow 0$ and if $\tau_{n, t} \rightarrow-\infty$. To see this, we first recall from Proposition 3.1 in [4] that (5.16) holds uniformly for $s_{n, t} \in(0, \infty)$ (or $\tau_{n, t}<0$ ) as $n \rightarrow \infty$, and it follows that the same holds for (5.17). Since $E$ is uniformly bounded, this implies that condition (b) of the $\mathrm{RH}$ problem for $R$ is uniform for $\tau_{n, t}<0$ as $n \rightarrow \infty$, and thus (5.20) is too.

\subsection{Proof of Theorem 1.7 for $t<0$}

If $\tau_{n, t} \rightarrow \tau<0$, we have by (5.10) that $s_{n, t} \rightarrow s \in(0,+\infty)$. We have that $s_{n, t}=4 n \pi \psi_{V}(0) \sqrt{-t}+$ $\mathcal{O}\left(n^{-1}\right)$ as $n \rightarrow \infty$.

First, recall formula (4.2) which expresses the correlation kernel in terms of $Y$. Expressing $Y$ in $T$ by (4.10), we obtain

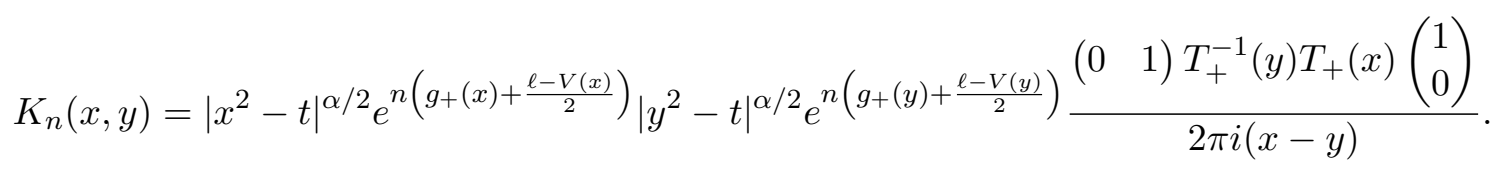


Using the identity $g_{+}-\frac{1}{2} V+\ell / 2=-\phi_{+}$on $(a, b)$ and substituting $S$ into the equation, by (5.1), we obtain

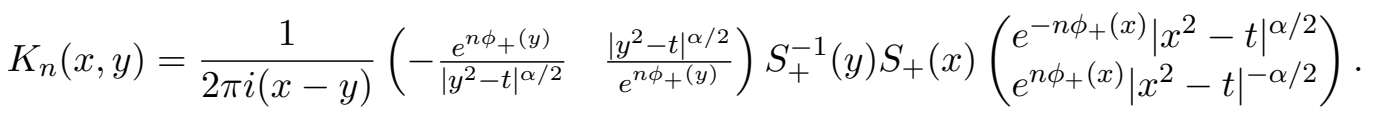

Now, we use the fact that $S=R P$ for $z$ close to the origin. Substituting this and the definition (5.11) of $P$, we obtain for $x, y \in U_{0}$ :

$$
\begin{aligned}
K_{n}(x, y)= & \frac{1}{2 \pi i(x-y)}\left(\begin{array}{ll}
1 & 1
\end{array}\right)\left(\Psi_{+}^{(1)}\right)^{-1}\left(\lambda(y) ; s_{n, t}\right) E_{+}^{-1}(y) \\
& \times R_{+}^{-1}(y) R_{+}(x) E_{+}(x) \Psi_{+}^{(1)}\left(\lambda(x) ; s_{n, t}\right)\left(\begin{array}{c}
1 \\
-1
\end{array}\right) .
\end{aligned}
$$

Up to this point, no approximations have been made. Now, we use the fact that $R=I+\mathcal{O}\left(n^{-1}\right)$ in the double scaling limit and the analyticity of $E$ and $R$, which imply that

$$
E_{+}^{-1}(y) R_{+}^{-1}(y) R_{+}(x) E_{+}(x)=I+\mathcal{O}(x-y),
$$

as $x \rightarrow y$ for $n$ sufficiently large. We find, for fixed $u, v$, that

$$
\begin{aligned}
& \frac{1}{\psi_{V}(0) n} K_{n, t}\left(x_{n}=\frac{u}{n \psi_{V}(0)}, y_{n}=\frac{v}{n \psi_{V}(0)}\right) \\
& \quad=\frac{1}{2 \pi i(u-v)}\left(\begin{array}{ll}
1 & 1
\end{array}\right)\left(\Psi_{+}^{(1)}\right)^{-1}\left(\lambda\left(y_{n}\right) ; s_{n, t}\right) \Psi_{+}^{(1)}\left(\lambda\left(x_{n}\right) ; s_{n, t}\right)\left(\begin{array}{c}
1 \\
-1
\end{array}\right)+\mathcal{O}\left(n^{-1}\right)
\end{aligned}
$$

as $n \rightarrow \infty, t \rightarrow 0$. By (2.3) and (2.42) we have

$$
\left(\begin{array}{c}
\Phi_{1}(\lambda ; \tau) \\
\Phi_{2}(\lambda ; \tau)
\end{array}\right)=e^{-\frac{s}{4} \sigma_{3}} \Psi^{(1)}(\lambda ; s=\sqrt{-\tau})\left(\begin{array}{c}
-1 \\
1
\end{array}\right),
$$

and it follows from (5.10) that

$$
\frac{1}{n \psi_{V}(0)} K_{n, t}\left(x_{n}=\frac{u}{n \psi_{V}(0)}, y_{n}=\frac{v}{n \psi_{V}(0)}\right)=\mathbb{K}_{\alpha}^{\mathrm{PV}}\left(\lambda\left(x_{n}\right), \lambda\left(y_{n}\right) ;-s_{n, t}^{2}\right)+\mathcal{O}\left(n^{-1}\right),
$$

as $n \rightarrow \infty, t \rightarrow 0$; with $\mathbb{K}_{\alpha}^{\mathrm{PV}}$ given in (1.27). In the case where $\tau_{n, t} \rightarrow \tau<0$,

$$
\lambda\left(x_{n}\right)=\pi u+\mathcal{O}\left(n^{-1}\right), \quad-s_{n, t}^{2}=\tau_{n, t}+\mathcal{O}\left(n^{-1}\right)
$$

which proves (1.26) by continuity of the kernel. If $\tau_{n, t} \rightarrow-\infty$, the error terms in (5.24) become of order $\mathcal{O}\left(n|t|^{3 / 2}\right.$ ). Nevertheless we can apply (1.28) (which holds whenever $u-v$ has a limit as $\tau \rightarrow-\infty)$ in (5.23) to obtain

$$
\lim _{s \rightarrow \infty} \mathbb{K}_{\alpha}^{\mathrm{PV}}\left(\lambda\left(x_{n}\right), \lambda\left(y_{n}\right) ;-s_{n, t}^{2}\right)=\mathbb{K}^{\sin }\left(\lambda\left(x_{n}\right), \lambda\left(y_{n}\right)\right)+\mathcal{O}\left(s_{n, t}^{-1}\right),
$$

as $n \rightarrow \infty, s_{n, t} \rightarrow \infty$. This yields (1.24), since $\lambda\left(x_{n}\right)-\lambda\left(y_{n}\right)=\pi(u-v)+\mathcal{O}\left(n^{-1}\right)$ as $n \rightarrow \infty$, uniformly in $t<t_{0}$ for some sufficiently small $t_{0}>0$. Finally, if $\tau_{n, t} \rightarrow 0$, we substitute (1.29) into (5.23) and obtain (1.25). This completes the proof of Theorem 1.7. 


\subsection{Proof of Theorem 1.1 for $t<0$}

We start from the differential identity (4.3). Following the transformations $Y \mapsto T \mapsto S \mapsto R$ given in (4.10), (5.1) and (5.19), and recalling the definition (5.11) of the local parametrix, we find the following form for $Y^{-1} Y^{\prime}$. Write $j=1$ for $\operatorname{Im} z>0$ and $j=2$ for $\operatorname{Im} z<0$. Then

$$
\begin{aligned}
\left(Y^{-1} Y^{\prime}\right)_{2,2}(z)= & \left(B(z)+W^{-1}(z) W^{\prime}(z)+e^{-n g(z) \sigma_{3}}\left(e^{n g(z) \sigma_{3}}\right)^{\prime}\right)_{2,2} \\
& +\left(\left(\left(\Psi^{(1)}\right)^{-1}\left(\Psi^{(1)}\right)_{z}^{\prime}\right)(\lambda(z))\right)_{j, j},
\end{aligned}
$$

where ()$^{\prime}$ in general means the derivative with respect to the main argument, ()$_{z}^{\prime}$ means the derivative with respect to $z$, and where

$$
B(z)=\left(\Psi^{(1)}(\lambda)\right)^{-1}(R E)^{-1}(z)(R E)^{\prime}(z) \Psi^{(1)}(\lambda) .
$$

We will show that $B(z)$ is bounded uniformly for $s \in(0, \infty)$ and $n$ large, but we will first calculate the other terms in the above expression. We have

$$
\left(\left(W^{-1} W^{\prime}\right)(z)+e^{-n g(z) \sigma_{3}}\left(e^{n g(z) \sigma_{3}}\right)^{\prime}\right)_{2,2}=-\frac{n}{2} V^{\prime}(z)+\frac{\alpha / 2}{z-z_{0}}+\frac{\alpha / 2}{z+z_{0}},
$$

where we used

$$
\phi_{ \pm}^{\prime}(x)+g_{ \pm}^{\prime}(x)=\frac{V^{\prime}}{2} .
$$

By condition (d) of the RH problem for $\Psi^{-}$and (2.42), it follows that

$$
\begin{aligned}
& \left(\left(\Psi^{(1)}\right)^{-1}\left(\Psi^{(1)}\right)_{z}^{\prime}\right)_{1,1}(\lambda(z)) \\
& \quad=-\frac{2 i \lambda^{\prime}\left(z_{0}\right)}{s_{n, t}}\left(H^{-1} H^{\prime}\right)_{1,1}(1)-\frac{\alpha / 2}{z-z_{0}}+\mathcal{O}(1) \quad \text { as } z \rightarrow z_{0},
\end{aligned}
$$

and

$$
\begin{aligned}
& \left(\left(\Psi^{(1)}\right)^{-1}\left(\Psi^{(1)}\right)_{z}^{\prime}\right)_{2,2}(\lambda(z)) \\
& \quad=-\frac{2 i \lambda^{\prime}\left(-z_{0}\right)}{s_{n, t}}\left(G^{-1} G^{\prime}\right)_{2,2}(0)-\frac{\alpha / 2}{z+z_{0}}+\mathcal{O}(1) \quad \text { as } z \rightarrow-z_{0},
\end{aligned}
$$

where both $\mathcal{O}(1)$ terms are uniform for large $n$ and sufficiently small $|t|$. Now, we use the following lemma, which we will prove below.

\section{Lemma 5.3.}

(1) The following identity holds:

$$
\left(G^{-1} G^{\prime}\right)_{2,2}(0)=-\left(H^{-1} H^{\prime}\right)_{1,1}(1)=\frac{s}{2}+\frac{\sigma_{\alpha}^{-}(s)}{\alpha}-\frac{\alpha}{2}
$$

(2) $B(z)$ is bounded uniformly as $n \rightarrow \infty$ for $t<t_{0}$ for some sufficiently small $t_{0}$.

Equations (5.25)-(5.28) and Lemma 5.3 can be substituted into (4.3) to obtain

$$
\begin{aligned}
\frac{d}{d t} \log \widehat{Z}_{n}(t, \alpha, V)= & -\frac{\alpha}{2 z_{0}}\left(\frac{4 i \lambda^{\prime}\left(z_{0}\right)}{s_{n, t}}\left(\frac{s_{n, t}}{2}+\frac{\sigma_{\alpha}^{-}\left(s_{n, t}\right)}{\alpha}-\frac{\alpha}{2}\right)+\frac{\alpha}{2 z_{0}}\right. \\
& \left.+\frac{n}{2}\left(V^{\prime}\left(-z_{0}\right)-V^{\prime}\left(z_{0}\right)\right)+B\left(z_{0}\right)-B\left(-z_{0}\right)+\mathcal{O}(1)\right)
\end{aligned}
$$

as $n \rightarrow \infty$ and for sufficiently small $|t|$. After a straightforward calculation in which one uses (1.11) and (5.10), Theorem 1.1 follows upon integration. 
Proof of Lemma 5.3. Equation (2.2) implies that

$$
\left(H^{-1} H^{\prime}\right)_{1,1}(1)=-\left(G^{-1} G^{\prime}\right)_{2,2}(0) \text {. }
$$

We proceed to evaluate $\left(G^{-1} G^{\prime}\right)_{2,2}(0)$. Substituting

$$
\Psi(\zeta)=G(\zeta) \zeta^{\frac{\alpha}{2} \sigma_{3}} \quad \text { for } \zeta \text { in a neighborhood of } 0,
$$

into (2.5), (2.7) and evaluating the terms of order $z^{-1}$, we find that

$$
\left(G \sigma_{3} G^{-1}\right)_{2,2}(0)=\frac{2 v}{\alpha}-1 .
$$

Writing

$$
G(z)=G_{0}\left(1+G_{1} z+\mathcal{O}\left(z^{2}\right)\right), \quad z \rightarrow 0,
$$

we also have

$$
\left(G^{-1} G^{\prime}\right)(0)=G_{1}
$$

and, by (2.8),

$$
\begin{aligned}
\frac{\partial}{\partial s} G_{0} & =B_{0} G_{0}, \\
\frac{\partial}{\partial s} G_{1} & =G_{0}^{-1} B_{1} G_{0}=-\frac{1}{2} G_{0}^{-1} \sigma_{3} G_{0} .
\end{aligned}
$$

By (5.30), (5.31) and (5.33), we obtain

$$
\frac{\partial}{\partial s}\left(G^{-1} G^{\prime}\right)_{2,2}(0)=-\frac{v}{\alpha}+\frac{1}{2} .
$$

By analyzing at the behavior of $\Psi$ as $s \rightarrow+\infty$ in Section 2.3, one finds that as $s \rightarrow+\infty$,

$$
\left(G^{-1} G^{\prime}\right)_{2,2}(0)=-\frac{\alpha}{2}+\frac{s}{2}+o(1) .
$$

Integrating (5.34) using (2.18) and comparing (5.35) to (1.11), one finds that

$$
\left(G^{-1} G^{\prime}\right)_{2,2}(0)=\frac{s}{2}+\frac{\sigma(s)}{\alpha}-\frac{\alpha}{2} .
$$

It remains to show that $B(z)$ is bounded. It follows from (5.20) and Remark 5.2 that

$$
R^{-1}(z) R^{\prime}(z)=\mathcal{O}\left(n^{-1}\right) \quad \text { as } n \rightarrow \infty, t \rightarrow 0 .
$$

We will show that $E$ and $E^{-1} E^{\prime}$ are bounded uniformly as $n \rightarrow \infty$ and $t \rightarrow 0$. For $z$ in a neighborhood of $z_{0}$, we write

$$
\begin{aligned}
& E(z)=\prod_{j=1}^{4} f_{j}(z), \\
& f_{1}(z)=D^{-1}(\infty) N_{0}(z), \\
& f_{2}(z)=D(z) \sigma_{1} \sigma_{3}\left(z+z_{0}\right)^{-\frac{\alpha}{2} \sigma_{3}}\left(\lambda(z)+\frac{i s_{n, t}}{4}\right)^{-\frac{\alpha}{2} \sigma_{3}} n^{\frac{\alpha}{2} \sigma_{3}},
\end{aligned}
$$




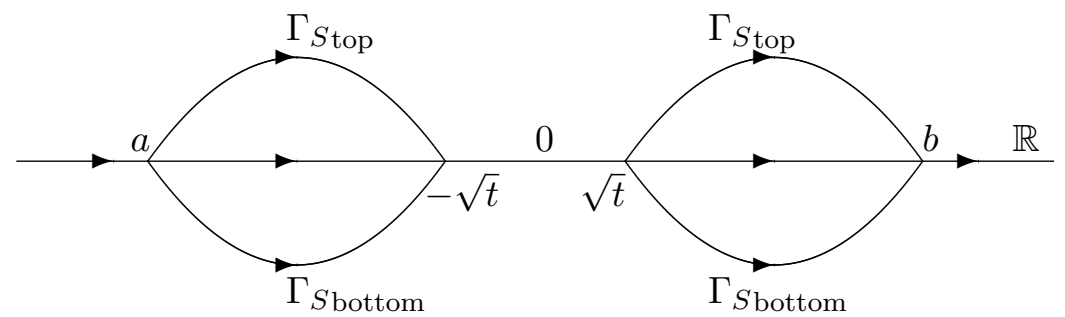

Figure 8. The contour $\Gamma_{S}$.

$$
\begin{aligned}
& f_{3}(z)=\left(z-z_{0}\right)^{-\frac{\alpha}{2} \sigma_{3}}\left(\lambda-\frac{i s_{n, t}}{4}\right)^{\frac{\alpha}{2} \sigma_{3}} n^{-\frac{\alpha}{2} \sigma_{3}}, \\
& f_{4}(z)=e^{n \phi(z) \sigma_{3}} e^{-i \lambda(z) \sigma_{3}} e^{\frac{\pi i \alpha}{2} \sigma_{3}} .
\end{aligned}
$$

Each $f_{j}, j=1, \ldots, 4$ is uniformly bounded at $\pm z_{0}$ as $n \rightarrow \infty$ and $t \rightarrow 0$. This is clear for $f_{1}$ and $f_{3}$. To see this for $f_{2}$, one can perform a contour integral to find that

$$
D\left(z_{0}\right)=\left(\begin{array}{cc}
\mathcal{O}\left(z_{0}^{-\alpha}\right) & 0 \\
0 & \mathcal{O}\left(z_{0}^{\alpha}\right)
\end{array}\right)
$$

For $f_{4}$, it follows from the definition of $\lambda$ and the fact that $\phi(z)=\overline{\phi(\bar{z})}$. One can do the same for $z$ in a neighborhood of $-z_{0}$. Thus

$$
B\left( \pm z_{0}\right)=\lim _{z \rightarrow \pm z_{0}}\left(\Psi^{(1)}\right)^{-1}\left(\lambda(z) ; s_{n, t}\right) \mathcal{O}(1) \Psi^{(1)}\left(\lambda(z) ; s_{n, t}\right)
$$

which is bounded as long as $s$ lies in compact subsets of $(0,+\infty)$. To see that $B\left( \pm z_{0}\right)$ is bounded as well as $s \rightarrow \infty$, we substitute in $\widehat{\Psi}$ from (2.40) and use the fact that it converges to the identity. To see that $B\left( \pm z_{0}\right)$ is bounded as $s \rightarrow 0$ we refer to the small $x$ behavior of $\Psi$ in [4] (formulas (4.23), (4.53), (4.61), (4.64), (4.67)).

\section{Asymptotic analysis of $T$ as $n \rightarrow \infty$ for $t>0$}

In this section, we will analyse asymptotically the $\mathrm{RH}$ problem for $T$ in the case where $t>0$, which means that the singularities $\pm \sqrt{t}$ are real and approach the origin as $t \rightarrow 0$. Many of the notations here will be the same as the ones in Section 5 to emphasize the parallels in the analysis. We will refer to many arguments given in the previous section, so it is recommended to read Section 5 before this one.

\subsection{Opening of the lens for $t>0$}

We open the lens as in Fig. 8. We define $S$ in the same way as for $t<0$ in (5.1), except that we use the contour pictured in Fig. 8 instead of the one in Fig. 7, and except for the fact that we define $\left(z^{2}-t\right)^{\alpha}$ such that it is positive on $(a,-\sqrt{t})$ and $(\sqrt{t}, b)$ :

$$
\left(z^{2}-t\right)^{\alpha}=\left|z^{2}-t\right|^{\alpha} e^{i \alpha \arg (z-\sqrt{t})} e^{i \alpha \arg (z+\sqrt{t})},
$$

with the conventions $-3 \pi / 2<\arg (z-\sqrt{t})<\pi / 2$ and $-\pi / 2<\arg (z+\sqrt{t})<3 \pi / 2$.

The RH problem for $S$ is the following. 


\section{RH problem for $S$}

(a) $S$ is analytic for $z \in \mathbb{C} \backslash \Gamma_{S}$ where $\Gamma_{S}=\Gamma_{S \text { top }} \cup \Gamma_{S \text { bottom }} \cup \mathbb{R}$ is the union of the lens and the real line, see Fig. 8.

(b) $S$ has the following jump relations on $\Gamma_{S}$,

$$
\begin{array}{ll}
S_{+}(x)=S_{-}(x)\left(\begin{array}{cc}
1 & \left|x^{2}-t\right|^{\alpha} e^{-2 n \phi(x)} \\
0 & 1
\end{array}\right) & \text { for } x<a \text { and } b<x, \\
S_{+}(x)=S_{-}(x)\left(\begin{array}{cc}
0 & \left|x^{2}-t\right|^{\alpha} \\
-\frac{1}{\left|x^{2}-t\right|^{\alpha}} & 0
\end{array}\right) & \text { for } x \in(a,-\sqrt{t}) \cup(\sqrt{t}, b), \\
S_{+}(z)=S_{-}(x)\left(\begin{array}{cc}
1 & 0 \\
\frac{e^{2 n \phi(z)}}{\left(z^{2}-t\right)^{\alpha}} & 1
\end{array}\right) & \text { for } z \in \Gamma_{S \text { top }} \cup \Gamma_{S \text { bottom }}, \\
S_{+}(x)=S_{-}(x)\left(\begin{array}{cc}
e^{2 n \phi_{+}(x)} & \left|x^{2}-t\right|^{\alpha} \\
0 & e^{2 n \phi_{-}(x)}
\end{array}\right) & \text { for } x \in[-\sqrt{t}, \sqrt{t}] .
\end{array}
$$

(c) $S(z)=I+\mathcal{O}\left(z^{-1}\right)$ as $z \rightarrow \infty$.

(d) For $\alpha<0, S$ has the following asymptotics as $z \rightarrow \pm \sqrt{t}$ :

$$
S(z)=\left(\begin{array}{ll}
\mathcal{O}(1) & \mathcal{O}\left(|z \mp \sqrt{t}|^{\alpha}\right) \\
\mathcal{O}(1) & \mathcal{O}\left(|z \mp \sqrt{t}|^{\alpha}\right)
\end{array}\right)
$$

For $\alpha \geq 0$ the following asymptotics hold:

$$
\begin{aligned}
& S(z)=\left(\begin{array}{ll}
\mathcal{O}(1) & \mathcal{O}(1) \\
\mathcal{O}(1) & \mathcal{O}(1)
\end{array}\right) \quad \text { as } z \rightarrow \pm \sqrt{t} \text { from outside the lense, } \\
& S(z)=\left(\begin{array}{ll}
\mathcal{O}\left(|z \mp \sqrt{t}|^{-\alpha}\right) & \mathcal{O}(1) \\
\mathcal{O}\left(|z \mp \sqrt{t}|^{-\alpha}\right) & \mathcal{O}(1)
\end{array}\right) \quad \text { as } z \rightarrow \pm \sqrt{t} \text { from inside the lense. }
\end{aligned}
$$

(e) $S(z)$ is bounded as $z \rightarrow a$ and as $z \rightarrow b$.

\subsection{Global parametrix}

As for $t<0$, we now ignore small neighborhoods of $a, b, 0$ and exponentially small jumps.

The solution to the RH problem obtained in this way is constructed as for $t<0$ : $N$ is given by (5.2)-(5.5), where the parameter $t$ is positive in (5.5).

The function $N$ now has singularities at $\pm \sqrt{t}$. Applying a contour deformation argument on the integral in (5.5), we obtain the following behavior,

$$
N(z)=\mathcal{O}\left(|z \pm \sqrt{t}|^{-\frac{\alpha}{2} \sigma_{3}}\right), \quad z \rightarrow \mp \sqrt{t} .
$$

\subsection{Local parametrices at endpoints $a$ and $b$}

The local parametrices at the endpoints $a$ and $b$ remain unchanged with respect to Section 5.3. They satisfy the same jumps as $S$ near $a$ and $b$, and as $n \rightarrow \infty$, they match with the global parametrix $N$ on the boundary of fixed disks around $a$ and $b$.

\subsection{Local parametrix at the origin}

Let $U_{0}$ be a sufficiently small disk around the origin which is independent of $n$. We will construct a function $P$ which satisfies the following conditions. 


\section{RH problem for $P$}

(a) $P: U_{0} \backslash \Gamma_{S} \rightarrow \mathbb{C}^{2 \times 2}$ is analytic.

(b) $P$ has same jump relations as $S$ :

$$
P_{-}^{-1}(z) P_{+}(z)=S_{-}^{-1}(z) S_{+}(z) \quad \text { for } z \in U_{0} \cap \Gamma_{S} .
$$

(c) As $n \rightarrow \infty$ and simultaneously $t \rightarrow 0$ in such a way that $n^{2} t$ tends to a non-zero constant, we have

$$
P(z)=\left(I+\mathcal{O}\left(n^{-1}\right)\right) N(z),
$$

uniformly for $z$ on the boundary $\partial U_{0}$.

These RH conditions are exactly the same as in the case $t<0$, but it has to be noted that the function $S$ and its jump contour are different here than in the case $t<0$. Therefore the construction of the local parametrix differs from the construction done before.

In analogy with the case $t<0$, define

$$
\lambda(z)=-i n \times \begin{cases}\phi(z)-\frac{\phi_{+}(\sqrt{t})+\phi_{+}(-\sqrt{t})}{2} & \text { for } \operatorname{Im} z>0, \\ -\phi(z)-\frac{\phi_{+}(\sqrt{t})^{2} \phi_{+}(-\sqrt{t})}{2} & \text { for } \operatorname{Im} z<0 .\end{cases}
$$

By (4.13), $\lambda$ is a conformal map in $U_{0}$,

$$
\lambda(\sqrt{t})=-\lambda(-\sqrt{t})=-\frac{i n}{2}\left(\phi_{+}(\sqrt{t})-\phi_{+}(-\sqrt{t})\right),
$$

and

$$
\lambda^{\prime}(0)=n \pi \psi_{V}(0) .
$$

We have that $\lambda(z)$ is a conformal map which sends $\sqrt{t}$ to $\left|s_{n, t}\right| / 4$ and $-\sqrt{t}$ to $-\left|s_{n, t}\right| / 4$, with

$$
s_{n, t}=-4 i \lambda(\sqrt{t})=-2 n\left(\phi_{+}(\sqrt{t})-\phi_{+}(-\sqrt{t})\right) .
$$

By (4.13), we have

$$
s_{n, t}=-2 \pi i n \int_{-\sqrt{t}}^{\sqrt{t}} \psi_{V}(s) d s=-4 i n \pi \sqrt{t} \psi_{V}(0)+\mathcal{O}\left(n t^{3 / 2}\right)=-i \sqrt{\tau_{n, t}}+\mathcal{O}\left(n t^{3 / 2}\right),
$$

as $t \rightarrow 0, n \rightarrow \infty$, similar to the case $t<0$.

Recall the definition of $\Psi^{(2)}$ in (3.11). We now fix the lens by requiring that it is mapped to the jump contours $\widetilde{\Gamma}_{1}, \widetilde{\Gamma}_{2}, \widetilde{\Gamma}_{3}$, and $\widetilde{\Gamma}_{4}$ of $\Psi^{(2)}$ by $\lambda$. We search for $P$ in the form

$$
P(z)=E(z) \Psi^{(2)}\left(\lambda(z) ; s_{n, t}\right) W(z),
$$

where

$$
W(z)= \begin{cases}\left(\left(z^{2}-t\right)^{\alpha / 2} e^{-n \phi(z)}\right)^{\sigma_{3}} \sigma_{3} \sigma_{1} & \text { for } \operatorname{Im} z>0, \operatorname{Re} \lambda(z) \notin\left[-\frac{\left|s_{n, t}\right|}{4}, \frac{\left|s_{n, t}\right|}{4}\right], \\ \left(\left(z^{2}-t\right)^{-\alpha / 2} e^{n \phi(z)}\right)^{\sigma_{3}} & \text { for } \operatorname{Im} z<0, \operatorname{Re} \lambda(z) \notin\left[-\frac{\left|s_{n, t}\right|}{4}, \frac{\left|s_{n, t}\right|}{4}\right], \\ \left(\left(z^{2}-t\right)^{\alpha / 2} e^{\frac{\pi i \alpha}{2}} e^{-n \phi(z)}\right)^{\sigma_{3}} \sigma_{3} \sigma_{1} & \text { for } \operatorname{Im} z>0, \operatorname{Re} \lambda(z) \in\left[-\frac{\left|s_{n, t}\right|}{4}, \frac{\left|s_{n, t}\right|}{4}\right], \\ \left(\left(z^{2}-t\right)^{-\alpha / 2} e^{-\frac{\pi i \alpha}{2}} e^{n \phi(z)}\right)^{\sigma_{3}} & \text { for } \operatorname{Im} z<0, \operatorname{Re} \lambda(z) \in\left[-\frac{\left|s_{n, t}\right|}{4}, \frac{\left|s_{n, t}\right|}{4}\right] .\end{cases}
$$


The above construction was done in such a way that $W$ induces the correct jump relations for $P$. $E$ is an analytic function which has to be such that the matching condition (c) of the RH problem for $P$ is valid for $z$ on the boundary of $U_{0}$. Therefore, we recall the definition of $\chi$ in $(3.12)$ and let

$$
E(z)=N(z) W^{-1}(z) e^{-i \lambda(z) \sigma_{3}} \chi(\lambda(z))^{-1} .
$$

Proposition 6.1. $P(z)$, defined as in (6.5), satisfies the $R H$ problem for $P$.

Proof. Condition (a) and condition (b) hold by construction. We proceed to prove (c). By the definition of $E(z)$, we have

$$
P(z) N^{-1}(z)=E(z) \Psi^{(2)}\left(\lambda(z) ; s_{n, t}\right) e^{-i \lambda(z) \sigma_{3}} \chi(\lambda(z))^{-1} E^{-1}(z) .
$$

For $z \in \partial U_{0}$, it follows from (3.13) that

$$
\Psi^{(2)}\left(\lambda(z) ; s_{n, t}\right)=\left(I+\mathcal{O}\left(n^{-1}\right)\right) \chi(\lambda(z)) e^{i \lambda(z) \sigma_{3}}
$$

as $n \rightarrow \infty$, where the $\mathcal{O}\left(n^{-1}\right)$ is uniform for $s_{n, t}$ in compact subsets of $(0,-i \infty)$ and $\lambda(z)$ sufficiently large. Using the same calculations as in the proof of Proposition 5.1 we find that $E$ is bounded on $\partial U_{0}$ and so the result follows in the same manner as in the proof of Proposition 5.1.

\subsection{Small norm RH problem}

A small norm RH problem can be constructed in a similar way as in Section 5.5: define

$$
R(z)= \begin{cases}S(z) P(z)^{-1} & \text { for } z \in U_{0}, \\ S(z) P^{(a)}(z)^{-1} & \text { for } z \in U_{a}, \\ S(z) P^{(b)}(z)^{-1} & \text { for } z \in U_{b}, \\ S(z) N(z)^{-1} & \text { for } z \in \mathbb{C} \backslash\left(U_{0} \cup U_{a} \cup U_{b}\right) .\end{cases}
$$

Then $R$ satisfies the following $\mathrm{RH}$ problem, similar to the case where $\tau<0$.

\section{RH problem for $\boldsymbol{R}$}

(a) $R$ is analytic on $\mathbb{C} \backslash \Gamma_{R}$ where

$$
\Gamma_{R}=\partial U_{a} \cup \partial U_{b} \cup \partial U_{0} \cup\left(\Gamma_{S} \backslash\left((a, b) \cup U_{0} \cup U_{a} \cup U_{b}\right)\right) .
$$

(b) $R$ has the following jump relations on $\Gamma_{R}$ :

$$
R_{+}(z)=R_{-}(z)\left(I+\mathcal{O}\left(n^{-1}\right)\right)
$$

as $n \rightarrow \infty$, uniformly for $z \in \Gamma_{R}$, in the double scaling limit where $\tau_{n, t} \rightarrow \tau>0$.

(c) $R(z)$ has the following asymptotics as $z \rightarrow \infty$ :

$$
R(z)=I+\mathcal{O}\left(z^{-1}\right) .
$$

In the double scaling limit where $n \rightarrow \infty$ and at the same time $t \rightarrow 0$ in such a way that $n^{2} t$ tends to a non-zero constant, it follows that

$$
R(z)=I+\mathcal{O}\left(n^{-1}\right),
$$

uniformly for $z$ off the jump contour for $R$. As for $t<0$, one can extend this result to the cases where $n \rightarrow \infty$ and $t \rightarrow 0$ in such a way that $n^{2} t \rightarrow \infty$ and in such a way that $n^{2} t \rightarrow 0$. We do not give the details here, as the arguments are very similar to those in [5]: see equations (5.1), (5.17), (5.18), (5.25) in [5] for $n^{2} t \rightarrow \infty$ and equations (6.1), (6.28), (6.32), and (4.6) in [5] for $n^{2} t \rightarrow 0$. 


\subsection{Proof of Theorem 1.7}

In the same way as when $t<0$, we can find an expression for the correlation kernel in terms of $S$ when $t>0$ :

$$
\begin{aligned}
K_{n}(x, y)= & \frac{1}{2 \pi i(x-y)}\left(-\frac{e^{n \phi_{+}(y)}}{\left|y^{2}-t\right|^{\alpha / 2}} \delta(y) \frac{\left|y^{2}-t\right|^{\alpha / 2}}{e^{n \phi_{+}(y)}}\right) \\
& \times S_{+}^{-1}(y) S_{+}(x)\left(\begin{array}{c}
\left|x^{2}-t\right|^{\alpha / 2} e^{-n \phi_{+}(x)} \\
e^{n \phi_{+}(x)}\left|x^{2}-t\right|^{-\alpha / 2} \delta(x)
\end{array}\right)
\end{aligned}
$$

where

$$
\delta(x)= \begin{cases}0 & \text { for } x \in(-\sqrt{t}, \sqrt{t}), \\ 1 & \text { for } x \in(a,-\sqrt{t}) \cup(\sqrt{t}, b) .\end{cases}
$$

Substituting the small norm RH solution $R$ into the formula for the correlation kernel in (6.7), one obtains

$$
\begin{aligned}
K_{n}(x, y)= & \frac{1}{2 \pi i(x-y)}\left(-\frac{e^{n \phi_{+}(y)}}{\left|y^{2}-t\right|^{\alpha / 2}} \delta(y) \frac{\left|y^{2}-t\right|^{\alpha / 2}}{e^{n \phi_{+}(y)}}\right) W_{+}^{-1}(y)\left(\Psi_{+}^{(2)}\right)^{-1}\left(\lambda(y) ; s_{n, t}\right) \\
& \times E_{+}^{-1}(y) R_{+}(y) R_{+}^{-1}(x) E(x) \Psi_{+}^{(2)}\left(\lambda(x) ; s_{n, t}\right) W(x)\left(\begin{array}{c}
e^{-n \phi_{+}(x)}\left|x^{2}-t\right|^{\alpha / 2} \\
e^{n \phi_{+}(x)}\left|x^{2}-t\right|^{-\alpha / 2} \delta(x)
\end{array}\right) .
\end{aligned}
$$

From the asymptotic behavior (6.6) and the analyticity of $R$ and $y$, we have

$$
E_{+}^{-1}(y) R_{+}(y) R_{+}(x) E(x)=I+\mathcal{O}(x-y),
$$

as $x \rightarrow y$ for $n$ sufficiently large. Substituting this into (6.9) along with the definition for $W$, one finds that

$$
\begin{aligned}
& K_{n}(x, y)=\frac{1}{2 \pi i(x-y)}(1 \quad \delta(y))\left(\Psi_{+}^{(2)}\right)^{-1}\left(\lambda(y) ; s_{n, t}\right) \\
& \times(I+\mathcal{O}(x-y)) \Psi_{+}^{(2)}\left(\lambda(x) ; s_{n, t}\right)\left(\begin{array}{c}
\delta(x) \\
-1
\end{array}\right) .
\end{aligned}
$$

Now, we can use (3.6) and (3.11) to express $\Psi^{(2)}$ in terms of the functions $\Phi_{1}$ and $\Phi_{2}$. We have the relation

$$
\left(\begin{array}{l}
\Phi_{1}(u ; \tau) \\
\Phi_{2}(u ; \tau)
\end{array}\right)=e^{-\frac{s}{4} \sigma_{3}} \begin{cases}\Psi_{+}^{(2)}(u ;-i \sqrt{\tau})\left(\begin{array}{c}
0 \\
-1
\end{array}\right), & -|s| / 4<u<|s| / 4 \\
\Psi^{(2)}(u ;-i \sqrt{\tau})\left(\begin{array}{c}
1 \\
-1
\end{array}\right), & u \in \mathbb{R} \backslash[-|s| / 4,|s| / 4]\end{cases}
$$

and after a similar calculation to the one in Section 5.6, using the fact that $\lambda^{\prime}(0)=\pi n \psi_{V}(0)$, one finds that Theorem 1.7 holds for $\tau_{n, t} \in(0, \infty)$.

\subsection{Proof of Theorem 1.1 for $t>0$}

We assume here that $\alpha>0$. The formulas (5.25), (5.26) which we obtained in the proof of Theorem 1.1 for $t<0$, hold also in the case $t>0$, if we replace $\Psi^{(1)}$ by $\Psi^{(2)}$. We obtain from (3.11) and condition d) in the $\mathrm{RH}$ problem for $\Psi^{+}$that as $z \rightarrow \sqrt{t}$,

$$
\left(\left(\Psi^{(2)}\right)^{-1}\left(\Psi^{(2)}\right)_{z}^{\prime}\right)_{2,2}(\lambda(z))=-\frac{\alpha / 2}{z-\sqrt{t}}+\frac{2 \lambda^{\prime}(z)}{|s|}\left(H^{-1} H^{\prime}\right)_{2,2}(1)+\mathcal{O}(1),
$$


where the error term is uniform for large $n$ and sufficiently small $t$. Likewise as $z \rightarrow-\sqrt{t}$,

$$
\left(\left(\Psi^{(2)}\right)^{-1}\left(\Psi^{(2)}\right)_{z}^{\prime}\right)_{2,2}(\lambda(z))=-\frac{\alpha / 2}{z+\sqrt{t}}+\frac{2 \lambda^{\prime}(z)}{|s|}\left(G^{-1} G^{\prime}\right)_{2,2}(0)+\mathcal{O}(1)
$$

where the error term is uniform for large $n$ and sufficiently small $t$. Thus, by (4.3), we obtain

$$
\begin{aligned}
\frac{d}{d t} \log \widehat{Z}_{n}(t, \alpha, V)= & -\frac{\alpha}{2 z_{0}}\left(\frac{2 i \lambda^{\prime}\left(z_{0}\right)}{s_{n, t}}\left(G^{-1} G^{\prime}\right)_{2,2}(0)-\frac{2 i \lambda^{\prime}\left(-z_{0}\right)}{s_{n, t}}\left(H^{-1} H^{\prime}\right)_{2,2}(1)\right. \\
& \left.+\frac{\alpha}{2 z_{0}}+\frac{n}{2}\left(V^{\prime}\left(-z_{0}\right)-V^{\prime}\left(z_{0}\right)\right)+B\left(z_{0}\right)-B\left(-z_{0}\right)+\mathcal{O}(1)\right)
\end{aligned}
$$

as $n \rightarrow \infty$ and sufficiently small $t$. As in the case $t<0$, we have

$$
\left(G^{-1} G^{\prime}\right)_{2,2}^{\prime}(0)=-\left(H^{-1} H^{\prime}\right)_{2,2}^{\prime}(1)=\frac{\sigma^{\prime}(s)}{\alpha}+\frac{1}{2},
$$

and we can use the large $s$ asymptotics given in [5, equation (5.1)] to find that

$$
\left(G^{-1} G^{\prime}\right)_{2,2}(0)=-\left(H^{-1} H^{\prime}\right)_{2,2}(1)=\frac{\sigma(s)}{\alpha}+\frac{s}{2}-\frac{\alpha}{2} .
$$

In the same manner as for $t<0$, one can show that

$$
B\left( \pm z_{0}\right)=\lim _{z \rightarrow \pm z_{0}}\left(\Psi^{(2)}\right)^{-1}\left(\lambda(z) ; s_{n, t}\right) \mathcal{O}(1) \Psi^{(2)}\left(\lambda(z) ; s_{n, t}\right)
$$

which is uniformly bounded for $n$ large and $s \in(0,-i \infty)$. To show this for $s \rightarrow-i \infty$, one uses equations (5.10) and (5.15) from [5]; while to show this for $s \rightarrow 0$, one uses equations (6.10), (6.19) and (6.23) from [5]. Substituting the formula for $G$ and $H$ into (6.11) with $B$ bounded yields Theorem 1.1 for $t, \alpha>0$ after integration.

\section{Acknowledgements}

The authors are grateful to I. Krasovsky and N. Simm for useful discussions. They were supported by the European Research Council under the European Union's Seventh Framework Programme (FP/2007/2013)/ ERC Grant Agreement 307074 and by the Belgian Interuniversity Attraction Pole P07/18.

\section{References}

[1] Akemann G., Dalmazi D., Damgaard P.H., Verbaarschot J.J.M., QCD 3 and the replica method, Nuclear Phys. B 601 (2001), 77-124, hep-th/0011072.

[2] Atkin M.R., A Riemann-Hilbert problem for equations of Painlevé type in the one matrix model with semi-classical potential, arXiv:1504.04539.

[3] Brézin E., Hikami S., Characteristic polynomials of random matrices, Comm. Math. Phys. 214 (2000), 111-135, math-ph/9910005.

[4] Claeys T., Its A., Krasovsky I., Emergence of a singularity for Toeplitz determinants and Painlevé V, Duke Math. J. 160 (2011), 207-262, arXiv:1004.3696.

[5] Claeys T., Krasovsky I., Toeplitz determinants with merging singularities, Duke Math. J. 164 (2015), 28972987, arXiv:1403.3639.

[6] Damgaard P.H., Nishigaki S.M., Universal massive spectral correlators and three-dimensional QCD, Phys. Rev. D 57 (1998), 5299-5302, hep-th/9711096. 
[7] Deift P., Orthogonal polynomials and random matrices: a Riemann-Hilbert approach, Courant Lecture Notes in Mathematics, Vol. 3, New York University, Courant Institute of Mathematical Sciences, New York, Amer. Math. Soc., Providence, RI, 1999.

[8] Deift P., Its A., Krasovsky I., Asymptotics of Toeplitz, Hankel, and Toeplitz+Hankel determinants with Fisher-Hartwig singularities, Ann. of Math. 174 (2011), 1243-1299, arXiv:0905.0443.

[9] Deift P., Its A., Krasovsky I., Toeplitz matrices and Toeplitz determinants under the impetus of the Ising model: some history and some recent results, Comm. Pure Appl. Math. 66 (2013), 1360-1438, arXiv:1207.4990.

[10] Deift P., Kriecherbauer T., McLaughlin K.T.-R., New results on the equilibrium measure for logarithmic potentials in the presence of an external field, J. Approx. Theory 95 (1998), 388-475.

[11] Deift P., Kriecherbauer T., McLaughlin K.T.-R., Venakides S., Zhou X., Strong asymptotics of orthogonal polynomials with respect to exponential weights, Comm. Pure Appl. Math. 52 (1999), 1491-1552.

[12] Deift P., Kriecherbauer T., McLaughlin K.T.-R., Venakides S., Zhou X., Uniform asymptotics for polynomials orthogonal with respect to varying exponential weights and applications to universality questions in random matrix theory, Comm. Pure Appl. Math. 52 (1999), 1335-1425.

[13] Deift P., Zhou X., A steepest descent method for oscillatory Riemann-Hilbert problems. Asymptotics for the MKdV equation, Ann. of Math. 137 (1993), 295-368, math.AP/9201261.

[14] Fokas A.S., Its A.R., Kapaev A.A., Novokshenov V.Yu., Painlevé transcendents: the Riemann-Hilbert approach, Mathematical Surveys and Monographs, Vol. 128, Amer. Math. Soc., Providence, RI, 2006.

[15] Fokas A.S., Its A.R., Kitaev A.V., The isomonodromy approach to matrix models in 2D quantum gravity, Comm. Math. Phys. 147 (1992), 395-430.

[16] Forrester P.J., Frankel N.E., Applications and generalizations of Fisher-Hartwig asymptotics, J. Math. Phys. 45 (2004), 2003-2028, math-ph/0401011.

[17] Forrester P.J., Frankel N.E., Garoni T.M., Witte N.S., Finite one-dimensional impenetrable Bose systems: occupation numbers, Phys. Rev. A 67 (2003), 043607, 17 pages, cond-mat/0211126.

[18] Forrester P.J., Witte N.S., Application of the $\tau$-function theory of Painlevé equations to random matrices: $\mathrm{P}_{\mathrm{VI}}$, the JUE, CyUE, cJUE and scaled limits, Nagoya Math. J. 174 (2004), 29-114, math-ph/0204008.

[19] Fyodorov Y.V., Khoruzhenko B.A., Simm N.J., Fractional Brownian motion with Hurst index $H=0$ and the Gaussian unitary ensemble, Ann. Probab., to appear, arXiv:1312.0212.

[20] Fyodorov Y.V., Simm N.J., On the distribution of the maximum value of the characteristic polynomial of GUE random matrices, arXiv:1503.07110.

[21] Garoni T.M., On the asymptotics of some large Hankel determinants generated by Fisher-Hartwig symbols defined on the real line, J. Math. Phys. 46 (2005), 043516, 19 pages, math-ph/0411019.

[22] Its A.R., Kuijlaars A.B.J., Östensson J., Critical edge behavior in unitary random matrix ensembles and the thirty-fourth Painlevé transcendent, Int. Math. Res. Not. 2008 (2008), no. 9, rnn017, 67 pages, arXiv:0704.1972.

[23] Jimbo M., Monodromy problem and the boundary condition for some Painlevé equations, Publ. Res. Inst. Math. Sci. 18 (1982), 1137-1161.

[24] Jimbo M., Miwa T., Studies on holonomic quantum fields. XVII, Proc. Japan Acad. Ser. A Math. Sci. 56 (1980), 405-410.

[25] Krasovsky I., Correlations of the characteristic polynomials in the Gaussian unitary ensemble or a singular Hankel determinant, Duke Math. J. 139 (2007), 581-619, math-ph/0411016.

[26] Kuijlaars A.B.J., McLaughlin K.T.-R., Generic behavior of the density of states in random matrix theory and equilibrium problems in the presence of real analytic external fields, Comm. Pure Appl. Math. 53 (2000), $736-785$

[27] Kuijlaars A.B.J., Vanlessen M., Universality for eigenvalue correlations at the origin of the spectrum, Comm. Math. Phys. 243 (2003), 163-191, math-ph/0305044.

[28] Muğan U., Fokas A.S., Schlesinger transformations of Painlevé II-V, J. Math. Phys. 33 (1992), 2031-2045.

[29] Olver F.W.J., Lozier D.W., Boisvert R.F., Clark C.W., NIST handbook of mathematical functions, U.S. Department of Commerce, National Institute of Standards and Technology, Washington, DC, Cambridge University Press, Cambridge, 2010. 
[30] Saff E.B., Totik V., Logarithmic potentials with external fields, Grundlehren der Mathematischen Wissenschaften, Vol. 316, Springer-Verlag, Berlin, 1997.

[31] Vanlessen M., Strong asymptotics of the recurrence coefficients of orthogonal polynomials associated to the generalized Jacobi weight, J. Approx. Theory 125 (2003), 198-237, math.CA/0212014.

[32] Wu T.T., McCoy B.M., Tracy C.A., Barouch E., Spin-spin correlation functions for the two-dimensional Ising model: exact theory in the scaling region, Phys. Rev. B 13 (1976), 316-374.

[33] Xu S.-X., Zhao Y.Q., Critical edge behavior in the modified Jacobi ensemble and Painlevé equations, Nonlinearity 28 (2015), 1633-1674, arXiv:1404.5105. 